\title{
Epidemiología de la interrelación cáncer colorrectal y diabetes mellitus tipo 2. Revisión sistemática
}

\author{
Epidemiology of the interrelationship between colorectal cancer and diabetes \\ mellitus type 2. Systematic review
}

\section{Epidemiologia da inter-relação câncer colorretal e Diabetes Mellitus tipo 2 - Revisão sistemática}

Deivis Javier Villanueva-Pájaro1; Edgar Ernesto Vergara-Dagobeth²; Amileth Suárez-Causado3; Rubén Darío Gómez-Arias4.

1 Doctor en Medicina Tropical (c), magíster en Inmunología, bacteriólogo, Grupo UNIMOL, Facultad de Medicina, Universidad de Cartagena. Cartagena, Colombia. deivisjavier.29@gmail.com. ORCID: orcid.org/0000-0001-5159-7976.

2 Doctor en Medicina Tropical, especialista en Salud Pública, especialista en Cirugía General, especialista en Cirugía de Mama y Tejidos Blandos, médico, investigador y líder del Grupo de Investigación Clínica en Medicina (GICLIM), Facultad de Medicina, Universidad de Sucre. Sincelejo, Colombia. edgar.vergara@unisucre.edu.co. oRCID: orcid.org/0000-0002-6962-7923.

3 Doctora en Bioquímica y Biología Molecular, especialista en Bioquímica Clínica, química farmaceuta, investigadora sénior Colciencias y líder del Grupo Prometeus \& Biomedicina Aplicada a las Ciencias Clínicas, Facultad de Medicina, Universidad de Cartagena. Cartagena, Colombia. asuarezc1@unicartagena.edu.co. ORCID: orcid.org/0000-0003-2807-0679.

4 Doctor en Salud Pública, magíster en Salud Pública, médico, investigador emérito Colciencias, investigador Facultad Nacional de Salud Pública, Universidad de Antioquia. Medellín, Colombia. rubengomez33@gmail.com. ORCID: orcid.org/0000-0002-4707-4862.

Recibido: 23/03/2019. Aprobado: 29/08/2019. Publicado: 20/03/2020

Villanueva-Pájaro DJ, Vergara-Dagobeth EE, Suárez-Causado A, Gómez-Arias RD. Epidemiología de la interrelación cáncer colorrectal y diabetes mellitus tipo 2. Revisión sistemática. Rev. Fac. Nac. Salud Pública. 2020;38(2):e337048. DOI: https://doi. org/10.17533/udea.rfnsp.e337048

\section{Resumen}

Objetivos: Describir el estado del arte sobre estudios epidemiológicos que evalúen asociaciones de riesgo entre el cáncer colorrectal y la diabetes tipo 2, y las políticas de salud pública internacionales y colombianas encaminadas a reducir tales patologías. Metodología: Revisión sistemática cualitativa sobre estudios epidemiológicos que evalúen asociaciones de riesgo entre el cáncer colorrectal y la diabetes tipo 2, publicados en idioma español e inglés en PubMed $^{\circledR}$, United States National Library of Medicine (U.S NLM ${ }^{\circledR}$ ), Embase ${ }^{\circledR}$, Literatura Latinoamericana y del Caribe en Ciencias de la Salud $\left(\right.$ Lilacs $\left.^{\circledR}\right)$ y Scientific Electronic Library Online (SciELO $\left.{ }^{\circledR}\right)$, antes del 30 de junio de 2019. Adicionalmente, se describen las políticas internacionales y nacionales para el control de la diabetes y el cáncer colorrectal. Resultados: De 251 estudios evaluados para elegibilidad, se incluyeron 32 investigaciones originales tipo casos y controles, cohorte prospectivo y retrospectivo, retrospectivos con enfoque de casos y controles, retrospectivos con enfoque casos-cohorte, al mostrar asociaciones de riesgo de padecer cáncer de colon o recto en individuos diabéticos tipo 2. Existen cinco normatividades internacionales para el control de diabetes y cáncer, y dos normatividades colombianas de control de ambas patologías. Conclusiones: La evidencia epidemiológica a nivel mundial señala que individuos con diabetes tipo 2 poseen mayor riesgo de padecer cáncer de 
colón o recto, en comparación con los no diabéticos, en una forma dependiente de la etnicidad y el sexo. Las políticas de salud pública internacionales y colombianas no han logrado reducir significativamente la carga de ambas patologías, ni los factores de riesgo comunes entre ambas condiciones, y tampoco reconocen los nexos epidemiológicos y biológicos entre la diabetes tipo 2 y el cáncer colorrectal.

--------Palabras clave: Neoplasias colorrectales; neoplasias del colon; neoplasias del recto; diabetes mellitus tipo 2; enfermedad crónica.

\section{Abstract}

Objective: Describe the state of the art on epidemiological studies that assess risk associations between colorectal cancer and type 2 diabetes, and Colombian and international public health policies to reduce these pathologies. Methodology: Qualitative systematic review on epidemiological studies that assess risk associations between colorectal cancer and type 2 diabetes, published in Spanish and English in PubMed $®$, United States National Library of Medicine (U.S NLM $\left.{ }^{\circledR}\right)$, Embase ${ }^{\circledR}$, Literatura Latinoamericana y del Caribe en Ciencias de la Salud (Lilacs ${ }^{\circledR}$ ) and Scientific Electronic Library Online (SciELO®), before 30 June 2019. Furthermore, we describe the international and national policies for the control of diabetes and colorectal cancer. Results: Of 251 studies assessed for eligibility, we included 32 original case-control studies, prospective and retrospective cohort studies, retrospective studies with a case-control approach and retrospective studies with a case-cohort approach as they showed risk associations for colon or rectal cancer in type 2 diabetic individuals. There are five international regulations for the control of diabetes and cancer, and two Colombian regulations for the control of both pathologies. Conclusions: The global epidemiological evidence indicates that individuals with type 2 diabetes have an increased risk of cancer of the colon or rectum, compared with non-diabetics, dependent on ethnicity and sex. International and Colombian public health policies have not been able to significantly reduce the burden of both pathologies, nor the common risk factors between both conditions, nor recognize the epidemiological and biological links between type 2 diabetes and colorectal cancer.

-Key words: Colorectal neoplasms; neoplasms of the colon; neoplasms of the rectum; diabetes mellitus type 2; chronic disease.

\section{Resumo}

Objetivo: Descrever o estado da arte relacionado aos estudos epidemiológicos que avaliam associações de risco entre o câncer colorretal e o diabetes tipo 2, e as políticas de saúde pública colombianas e internacionais encaminhadas à redução de tais patologias. Metodologia: Revisão sistemática qualitativa sobre estudos epidemiológicos que avaliem associações de risco entre o câncer colorretal e o diabetes tipo 2, publicados nos idiomas espanhol e inglês em PubMed®, United States National Library of Medicine (U.S NLM $\AA$ ), Embase ${ }^{\circledR}$, Literatura Latinoamericana y del Caribe em Ciencias de la Salud (Lilacs ${ }^{\circledR}$ ) e Scientific Electronic Library Online (SciELO®), antes de 30 de junho de 2019. Adicionalmente são descritas as políticas nacionais e internacionais para o controle do diabetes e o câncer colorretal. Resultados: Dos 251 estudos avaliados para elegibilidade foram incluídas 32 pesquisas originais tipo casos e controles, coorte prospectivo e retrospectivo, retrospectivos com foco de casos e controles, retrospectivos com foco casos-coorte, ao mostrar associações de risco de sofrer câncer de cólon ou reto em pacientes diabéticos tipo 2. Existem cinco regulamentos internacionais para o controle de diabetes e câncer e dois regulamentos colombianos de controle para ambas as patologias. Conclusões: A evidência epidemiológica a nível mundial mostra que pacientes com diabetes tipo 2 possuem um risco maior de sofrer câncer de cólon ou reto, em comparação com os não-diabéticos, numa forma dependendo da etnia e do sexo. As políticas de saúde pública colombianas e internacionais ainda não conseguiram diminuir significativamente a carga de ambas as patologias, nem os fatores de risco comuns entre ambas as condições, além de não reconhecerem os nexos epidemiológicos e biológicos entre o diabetes tipo 2 e o câncer colorretal.

Palavras-chave: Neoplasias colorretais; neoplasias do cólon; neoplasias do reto; diabetes Mellitus tipo 2; doença crônica.

\section{Introducción}

El cáncer y la diabetes son enfermedades crónicas, consideradas por la Organización Mundial de la Salud (oms) como problemas prioritarios de salud pública mundial [1]. La Agencia Internacional para la Investigación del Cáncer (IARC) estimó la incidencia mundial del cáncer para 2018 en 18,1 millones, en todas las edades y sexos combinados: 9,5 millones en hombres y 8,6 millones en mujeres, acompañado de 9,6 millones de muertes: 5,4 millones en hombres y 4,2 millones en mujeres. Las regiones mundiales que más contribuyeron al incremento de tales cifras son, en su orden: Asia $(48,4 \%)$, Europa $(23,4 \%)$ y las Américas $(21 \%)$ para incidencia, y Asia (57,3 \%), Europa (20,3\%) y las Américas/Caribe (14,4\%) para mortalidad [1]. 
Actualmente, el cáncer se posiciona como la segunda causa de muerte mundial, así como la primera o segunda causa de muerte prematura en 91 de 172 países, y como la tercera o cuarta en otros 22 países [2]. El riesgo acumulado y la probabilidad de morir prematuramente por cáncer para ambos sexos son 21,4 y $17,7 \%$, respectivamente; es decir, en 2018,1 de cada 5 hombres y 1 de cada 6 mujeres padecieron algún tipo de cáncer, y 1 de cada 8 hombres y 1 de cada 10 mujeres fallecieron por esta enfermedad [1].

En concordancia con lo anterior, el Global Burden of Disease Cancer señala que, en las dos últimas décadas, la tasa de mortalidad por cáncer creció $57 \%$ a nivel mundial [2]. Asimismo, IARC proyectó, para el 2032, un incremento general del $81 \%$ en la incidencia mundial del cáncer, es decir, 22 millones de nuevos casos, representados principalmente por adultos de países de alto y muy alto índice de desarrollo humano [3].

Por su parte, el cáncer colorrectal ( $\mathrm{CCR}$ ) representa, en la actualidad, la tercera causa en incidencia y la segunda en mortalidad en ambos sexos a nivel mundial, siendo más frecuente en hombres [1]. Igualmente, se prevé que la carga global para CCR en 2030 superará los 2,2 millones de nuevos casos, acompañado de 1,1 millones de muertes, lo cual representaría un crecimiento del $60 \%$ en la incidencia del CCR en tan solo veinte años [4].

Del mismo modo, Latinoamérica ha tendido al aumento en la incidencia y la mortalidad general atribuible a cáncer [4] y en la mortalidad general específica de CCR durante las dos últimas décadas [5]. El número actual de afectados por cáncer en Latinoamérica y el Caribe asciende a 2,6 millones, y proyecciones para 2035 indican que se presentarán 135 mil nuevos casos de CCR en todas las edades y sexos combinados: 66 mil en hombres y 62 mil en mujeres [6].

En relación con Colombia, el CCR se ubicó tercero en incidencia y en mortalidad para 2018, siendo mayor su frecuencia en mujeres [1]. De hecho, se esperan 12732 nuevos casos de CCR en todas las edades y sexos para 2035 en el país: 6805 en mujeres y 5927 en hombres [7]. El anterior panorama se agrava tras considerar que en Colombia, la mayoría de casos de CCR son diagnosticados y tratados en estadios avanzados de la enfermedad [8].

En forma similar, la incidencia de diabetes se ha cuadruplicado a nivel global durante las tres últimas décadas. Dicho incremento está representado especialmente por la diabetes mellitus tipo 2 (DMT2) [9]. En 2017, se reportaron 424,9 millones de personas con diabetes y 1,8 millones de defunciones atribuibles a esta enfermedad [10], de las cuales el $80 \%$ habitaban en países de bajos y medianos ingresos económicos, en su mayoría de edad productiva [11].

La cifra actual de individuos diabéticos en Centroamérica y Suramérica asciende a 29,6 millones, fuera de las cuales 11,5 millones conviven con diabetes sin recibir diagnóstico. El crecimiento esperado en la incidencia de DMT2 en adultos de países en desarrollo y desarrollados entre 2010 y 2030 se ha estimado en 69 y $20 \%$, respectivamente [10,11]. Así mismo, se espera un aumento del 148 y el $65 \%$ en la incidencia de diabetes en Latinoamérica y Centro/Suramérica respectivamente, durante el mismo periodo [12].

En Colombia, la diabetes se ubicó entre las tres primeras causas de mortalidad entre 2005 y 2014, y algunos estudios indican que su prevalencia actual en población general es cercana al $9 \%$, siendo mayor en individuos de áreas urbanas que rurales, y en mujeres [13]. En 2030, la población colombiana exhibirá un incremento del $54 \%$ en la incidencia de diabetes [12].

$\mathrm{El}$ anterior escenario permite interrogarnos sobre la influencia que pudiera ejercer la diabetes tipo 2 como factor de riesgo para el desarrollo del cáncer colorrectal. Por tanto, el objetivo de la presente revisión sistemática cualitativa es describir la evidencia epidemiológica que evalúe la diabetes tipo 2 como factor de riesgo para el desarrollo del cáncer colorrectal a nivel mundial, así como las políticas de salud pública internacionales y colombianas para el control de ambas patologías.

\section{Metodología}

\section{Pregunta de investigación}

¿Es la diabetes mellitus tipo 2 un factor de riesgo para el desarrollo del cáncer colorrectal en la población adulta a nivel mundial? Y si esto resultara cierto, ¿existen políticas de salud pública eficaces para controlar este riesgo?

\section{Fuentes de datos y estrategia de búsqueda}

La presente revisión sistemática es cualitativa, sobre factores de riesgo, e incluyó estudios epidemiológicos originales que evaluaron el riesgo de desarrollar cáncer de colon o de recto en adultos de ambos sexos con diagnóstico de DMT2, a nivel mundial. Fueron seguidas las declaraciones PRISMA (Preferred Reporting Items for Systematic reviews and Meta-Analyses) [14]. Estas guías son ampliamente utilizadas a nivel internacional, por cuanto permiten a los autores mejorar la presentación y la calidad crítica de sus revisiones sistemáticas y metaanálisis. Sin embargo, no es un instrumento para valorar la calidad de las revisiones sistemáticas.

Dos investigadores, de manera independiente, realizaron una primera búsqueda estructurada de publicaciones presentes en la web antes del 30 de junio de 2019, en idioma inglés y español, en PubMed ${ }^{\circledR}$, United States National Library of Medicine (U.S NLM ${ }^{\circledR}$ ), Embase ${ }^{\circledR}$, Literatura Latinoamericana y del Caribe en Ciencias de la Salud $\left(\right.$ Lilacs $\left.^{\circledR}\right)$ y Scientific Electronic Library Online 
(SciELO)_. Los términos utilizados en la primera búsqueda incluyeron los siguientes Descriptores en Ciencias de la Salud (DeCS): "Type 2 diabetes (Diabetes Tipo 2)", "Diabetes Mellitus Type 2 (Diabetes Mellitus Tipo 2)", "Colorectal Cancer (Cáncer colorrectal)", "Colorectal Neoplasms (Neoplasia colorrectal)", "Colonic Neoplasms (Neoplasia del colon)" y "Rectal Neoplasms (Neoplasia del recto)". Los documentos así obtenidos fueron añadidos al gestor bibliográfico EndNote y filtrados para comparar los duplicados según título, autores, resumen y número de páginas.

Luego, se seleccionaron las publicaciones no duplicadas, entre las que se realizó una segunda búsqueda de estudios epidemiológicos que evaluaran el riesgo de desarrollar CCR en personas adultas con diagnóstico de DMT2 a nivel mundial. Los términos utilizados en la segunda búsqueda incluyeron los siguientes descriptores DeCS: "Type 2 Diabetes AND Colorectal Cancer", y
"Diabetes tipo 2 y Cáncer colorrectal". De esta segunda búsqueda se escogieron los estudios de investigación original relacionados con la pregunta de investigación. Finalmente, se revisaron completamente uno a uno los contenidos de los estudios evaluados para elegibilidad y se aplicaron los criterios de inclusión y exclusión.

Al mismo tiempo, otros dos investigadores hicieron la revisión de las políticas internacionales y colombianas para el control de la diabetes y el cáncer colorrectal, disponibles en el índice bibliográfico de revistas indexadas del Departamento Administrativo de Ciencia, Tecnología e Innovación de Colombia (Colciencias).

La escogencia de los idiomas en los términos de búsqueda obedeció al interés de tamizar el mayor número de publicaciones relacionadas con la pregunta de investigación y, a su vez, facilitar la identificación de estudios en población hispanoamericana.

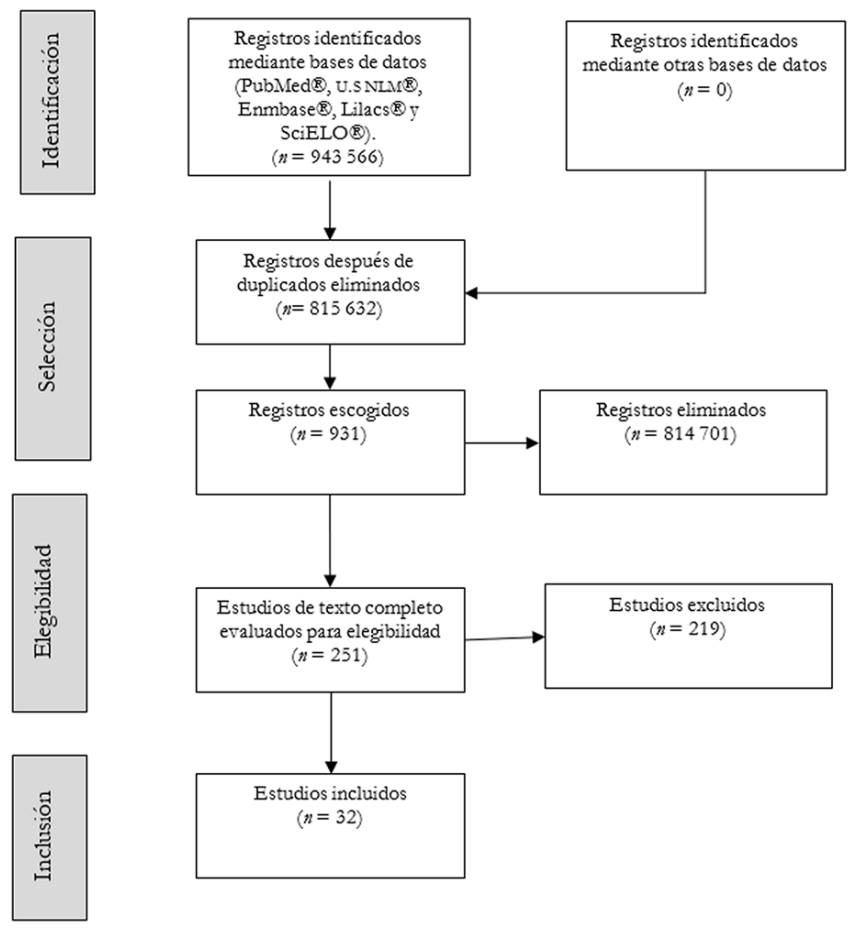

Figura 1. Flujograma del proceso de selección de los textos Fuente: [14].

El resumen de los pasos seguidos para la selección de estudios se muestra en la figura 1 .

\section{Selección de estudios y criterios de inclusión y de exclusión}

Se incluyeron estudios de investigación original tipo casos y controles, estudios de cohorte prospectiva, cohorte retrospectiva, retrospectivos con enfoque de casos y controles, retrospectivos con enfoque casoscohorte, que evaluaron el riesgo de desarrollar cáncer de colon o de recto en adultos de ambos sexos con diagnóstico de DMT2, a nivel mundial.

Los criterios de inclusión y exclusión de los estudios se presentan en la tabla 1 . 
Tabla 1. Criterios de inclusión y exclusión de estudios epidemiológicos.

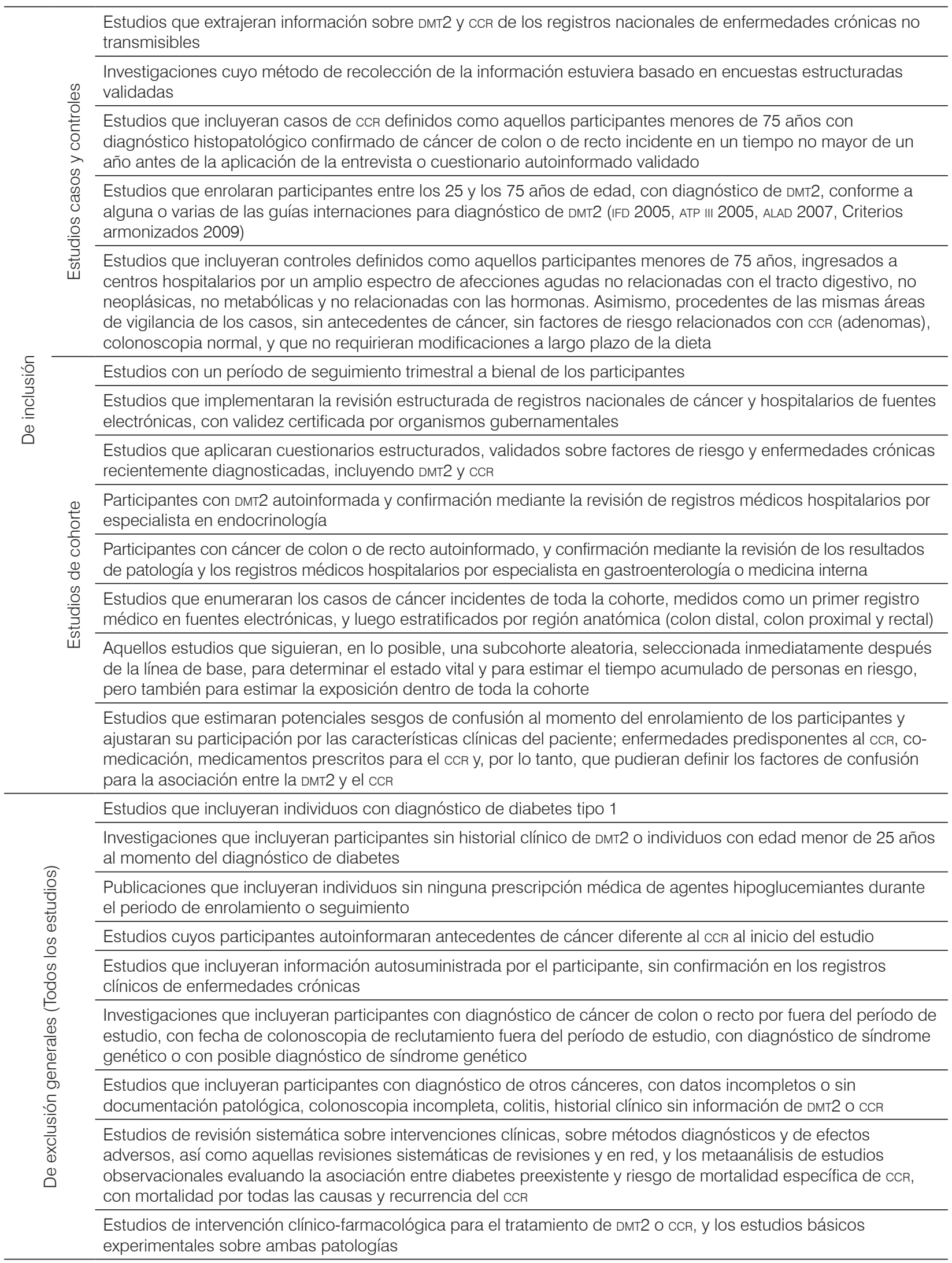

CCR: Cáncer colorrectal; DMT2: Diabetes mellitus tipo 2 


\section{Extracción de datos y evaluación de la calidad}

La extracción de los datos fue realizada independientemente por dos investigadores, de forma manual, mediante el empleo de una hoja de extracción. Los datos de interés consistieron en título de publicación, tipo de estudio, población, metodología, resultados y cumplimiento de criterios de inclusión.

Tabla 2. Estudios epidemiológicos sobre la interrelación diabetes mellitus tipo 2 y cáncer de colon o recto. Evaluación de la calidad de estudios según recomendaciones Newcastle-Ottawa [15]

\begin{tabular}{|c|c|c|c|c|}
\hline $\begin{array}{c}\text { Autor, año } \\
\text { publicación, } \\
\text { referencia }\end{array}$ & $\begin{array}{c}\text { Población, } \\
\text { período de } \\
\text { estudio o } \\
\text { seguimiento }\end{array}$ & $\begin{array}{c}\text { Tamaño muestra y tipo de } \\
\text { estudio }\end{array}$ & Hallazgos principales & $\begin{array}{l}\text { Nivel de } \\
\text { calidad }\end{array}$ \\
\hline $\begin{array}{l}\text { La Vecchia et al., } \\
1991[16]\end{array}$ & $\begin{array}{l}\text { Italia, ambos } \\
\text { sexos, 1985- } \\
1990\end{array}$ & $\begin{array}{l}\text { Casos y controles } \\
(n=2579) \\
673 \text { casos cáncer de colon. } \\
405 \text { casos cáncer de recto. } \\
1501 \text { controles. }\end{array}$ & $\begin{array}{l}\text { El historial clínico de DMT2 se asoció } \\
\text { significativamente con el riesgo de } \\
\text { desarrollar cáncer de colon (RR: 1,6, } \\
\text { Ic95 \%: } 1,1-2,3) \text {, mas no con cáncer } \\
\text { de recto (RR: 1,3, Ic95 \%: 0,8-2,0). } \\
\text { Test Mantel-Haenszel }\end{array}$ & $\begin{array}{l}\text { Bueno } \\
\text { Selección (4); } \\
\text { Comparabilidad } \\
\text { (1); } \\
\text { Exposición (3) }\end{array}$ \\
\hline $\begin{array}{l}\text { Steenland et al., } \\
1995[17]\end{array}$ & $\begin{array}{l}\text { Estados } \\
\text { Unidos, ambos } \\
\text { sexos, 1970- } \\
1987\end{array}$ & $\begin{array}{l}\text { Cohorte retrospectiva } \\
(n=4407) \\
176 \text { casos incidentes de } \\
\text { CCR: } 94 \text { en hombres y } 82 \text { en } \\
\text { mujeres }\end{array}$ & $\begin{array}{l}\text { El historial clínico de DMT2 se asoció } \\
\text { con el riesgo de desarrollar CCR en } \\
\text { hombres (OR: 1,43, IC95 \%: 0,61- } \\
3,31 \text { ) y en mujeres (OR: 1,40; IC95 \%: } \\
0,64-3,10 \text { ). Regresión de Cox }\end{array}$ & $\begin{array}{l}\text { Bueno } \\
\text { Selección (4); } \\
\text { Comparabilidad } \\
\text { (1); } \\
\text { Desenlace (3) }\end{array}$ \\
\hline $\begin{array}{l}\text { La Vecchia et al., } \\
1997 \text { [18] }\end{array}$ & $\begin{array}{l}\text { Italia, ambos } \\
\text { sexos, 1992- } \\
1996\end{array}$ & $\begin{array}{l}\text { Casos y controles } \\
\text { multicéntrico } \\
(n=6107) \\
1225 \text { casos de cáncer de } \\
\text { colon. } \\
728 \text { casos de cáncer de recto. } \\
4154 \text { controles }\end{array}$ & 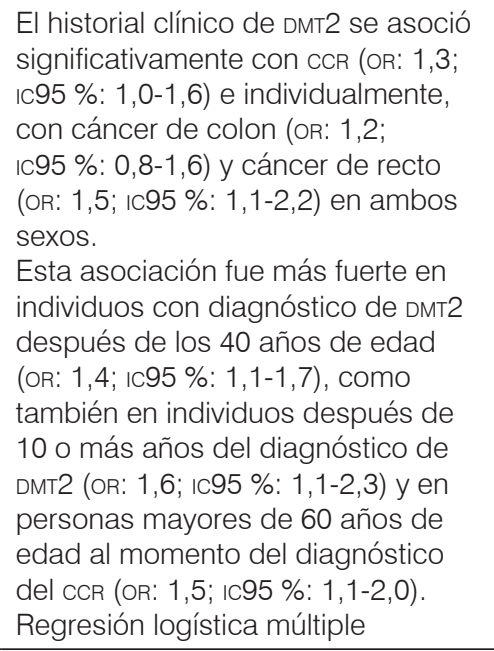 & $\begin{array}{l}\text { Bueno } \\
\text { Selección (4); } \\
\text { Comparabilidad } \\
(1) ; \\
\text { Exposición (3) }\end{array}$ \\
\hline $\begin{array}{l}\text { Le Marchand et } \\
\text { al., } 1997 \text { [19] }\end{array}$ & $\begin{array}{l}\text { Estados } \\
\text { Unidos, } \\
\text { multiétnico, } \\
\text { ambos sexos, } \\
1987-1991\end{array}$ & $\begin{array}{l}\text { Casos y controles } \\
(n=2384) \\
1192 \text { casos. } \\
1192 \text { controles. }\end{array}$ & $\begin{array}{l}\text { El historial de DMT2 se asoció con } \\
\text { cáncer de colon en lado izquierdo } \\
\text { en mujeres (OR: 3,0; IC95 \%: 1,2-7,1) } \\
\text { y hombres (OR: 1,9; IC95 \%: 1,1-3,5), } \\
\text { y en recto solo en mujeres (OR: 1,7; } \\
\text { IC95\%: 0,7-4,4). Regresión logística }\end{array}$ & $\begin{array}{l}\text { Bueno } \\
\text { Selección (4); } \\
\text { Comparabilidad } \\
(1) ; \\
\text { Exposición (3) }\end{array}$ \\
\hline $\begin{array}{l}\text { Hu et al., } 1999 \\
\text { [20] }\end{array}$ & $\begin{array}{l}\text { Estados } \\
\text { Unidos, solo } \\
\text { mujeres, 1976- } \\
1994\end{array}$ & $\begin{array}{l}\text { Cohorte prospectiva } \\
\text { (n=18 403) } \\
892 \text { casos incidentes de ccr: } \\
607 \text { casos cáncer de colon. } \\
176 \text { casos cáncer de recto. } \\
109 \text { casos sin subsitio } \\
\text { específico de ccr. }\end{array}$ & $\begin{array}{l}\text { El historial clínico de DMT2 se asoció } \\
\text { con incremento en el riesgo de } \\
\text { padecer CCR (RR ajustado: } 1,43 \text {, } \\
\text { Ic95 \%: 1,1-1,87, } p=0,009 \text { ) y de } \\
\text { cáncer de colon (RR ajustado: } 1,49 \text {, } \\
\text { Ic95 \%: 1,09-2,06, } p=0,01 \text { ), más } \\
\text { no con cáncer de recto (RR ajustado: } \\
\text { 1,11, Ic95\%: 0,56-2,21, } p=0,76 \text { ). } \\
\text { La DMT2 se asoció con CCR de } \\
\text { estadio avanzado (RR ajustado: 1,56, } \\
\text { Ic95 \%: 1,07-2,28, } p=0,02) \text {, como } \\
\text { también, con CCR fatal (RR ajustado: } \\
\text { 2,39, IC95 \%: } 1,46-3,92, p=0,0005 \text { ). }\end{array}$ & $\begin{array}{l}\text { Bueno } \\
\text { Selección (4); } \\
\text { Comparabilidad } \\
\text { (1); } \\
\text { Desenlace (3) }\end{array}$ \\
\hline
\end{tabular}




\begin{tabular}{|c|c|c|c|c|}
\hline $\begin{array}{l}\text { Autor, año } \\
\text { publicación, } \\
\text { referencia }\end{array}$ & $\begin{array}{l}\text { Población, } \\
\text { período de } \\
\text { estudio o } \\
\text { seguimiento }\end{array}$ & $\begin{array}{c}\text { Tamaño muestra y tipo de } \\
\text { estudio }\end{array}$ & Hallazgos principales & $\begin{array}{l}\text { Nivel de } \\
\text { calidad }\end{array}$ \\
\hline & & & $\begin{array}{l}\text { En mujeres padeciendo DMT2 } \\
\text { durante } 11 \text { a } 15 \text { años hubo } \\
\text { mayor asociación con CCR (RR } \\
\text { ajustado: } 2,39 \text {, Ic95 \%: } 1,43-3,71 \text {, } \\
p=0,0006 \text { ), con cáncer de } \\
\text { colon (RR ajustado: } 2,83 \text {, Ic95 \%: } \\
\text { 1,67- 4,78, } p=0,0001 \text { ), con CCR } \\
\text { avanzado (RR ajustado: } 2,25 \text {, Ic95 \%: } \\
1,11-4,58, p=0,02 \text { ) y con cCR fatal } \\
\text { (RR ajustado: } 3,96 \text {, Ic95 \%: } 1,72- \\
9,12, p=0,001 \text { ). Regresión logística } \\
\text { multivariada }\end{array}$ & \\
\hline $\begin{array}{l}\text { Nilsen et al., } \\
2001 \text { [21] }\end{array}$ & $\begin{array}{l}\text { Noruega, } \\
\text { ambos sexos, } \\
\text { 1984-1996 }\end{array}$ & $\begin{array}{l}\text { Cohorte prospectiva } \\
(n=75219) \\
730 \text { casos incidentes de CCR }\end{array}$ & $\begin{array}{l}\text { Mujeres con historial de DMT2 } \\
\text { poseen un } 55 \% \text { de mayor riesgo } \\
\text { de padecer CCR (RR ajustado: 1,55; } \\
\text { Ic95\%; 1,04-2,31) versus mujeres } \\
\text { no diabéticas. La anterior asociación } \\
\text { permaneció positiva para cáncer de } \\
\text { colon (RR: 1,6; IC95 \%: 1,02-2,51) y } \\
\text { cáncer de recto (RR: 1,41; Ic95 \%: } \\
\text { 0,61-3,27) en análisis individuales } \\
\text { por sitio de cáncer. Regresión de } \\
\text { Cox. } \\
\text { No se detectó asociación entre DMT2 } \\
\text { y cCR en hombres (RR: 0,66; IC95 \%: } \\
0,35-1,24 \text { ) }\end{array}$ & $\begin{array}{l}\text { Bueno } \\
\text { Selección (4); } \\
\text { Comparabilidad } \\
\text { (1); } \\
\text { Desenlace (3) }\end{array}$ \\
\hline $\begin{array}{l}\text { Limburg et al., } \\
2005 \text { [22] }\end{array}$ & $\begin{array}{l}\text { Estados } \\
\text { Unidos, solo } \\
\text { mujeres, 1987- } \\
1999\end{array}$ & $\begin{array}{l}\text { Cohorte prospectiva } \\
(n=34972) \\
1900 \text { mujeres con DMT2. } \\
870 \text { casos incidentes de CCR }\end{array}$ & $\begin{array}{l}\text { Mujeres posmenopáusicas con } \\
\text { DMT2 exhibieron un incremento } \\
\text { significativo en el riesgo de padecer } \\
\text { CCR (RR: } 1,5 ; \text { ic95 \%: 1,2-2,0). } \\
\text { Análisis por subsitio anatómico } \\
\text { mostró un fuerte incremento en el } \\
\text { riesgo de padecer cáncer en colon } \\
\text { proximal (RR: } 1,9 \text {; Ic95 \%: 1,3-2,6), } \\
\text { mas no así para cáncer en colon } \\
\text { distal (RR: } 1,1 ; \text { Ic95 \%: 0,6-1,8) ni } \\
\text { en recto (RR: 0,8; IC95 \%: 0,4-1,6). } \\
\text { Regresión de Cox }\end{array}$ & $\begin{array}{l}\text { Bueno } \\
\text { Selección (4); } \\
\text { Comparabilidad } \\
\text { (1); } \\
\text { Desenlace (3) }\end{array}$ \\
\hline $\begin{array}{l}\text { Yang et al., } 2005 \\
\text { [23] }\end{array}$ & $\begin{array}{l}\text { Reino Unido, } \\
\text { ambos sexos, } \\
\text { 1987-2002 }\end{array}$ & $\begin{array}{l}\text { Casos y controles } \\
(n=114876) \\
10447 \text { casos de ccr. } \\
104429 \text { controles. }\end{array}$ & $\begin{array}{l}\text { En individuos de ambos sexos, el } \\
\text { diagnóstico de DMT2 se asoció como } \\
\text { factor de riesgo para el desarrollo } \\
\text { de cCR (OR: 1,42; Ic95 \%: 1,25-1,62). } \\
\text { Tanto en mujeres (OR: 1,38; IC95 \%: } \\
\text { 1,14-1,67) como en hombres (OR: } \\
\text { 1,36; IC95 \%: 1,16-1,61) se detectó } \\
\text { asociación entre la DMT2 como factor } \\
\text { de riesgo para el desarrollo de cCR. } \\
\text { El estatus de DmT2 incrementó el } \\
\text { riesgo de cáncer de colon (OR: } 1,45 \text {; } \\
\text { Ic95 \%: 1,25-1,7) y de recto (OR: } \\
\text { 1,34; Ic95 \%: 1,08-1,68). Regresión } \\
\text { logística }\end{array}$ & $\begin{array}{l}\text { Bueno } \\
\text { Selección (4); } \\
\text { Comparabilidad } \\
(1) ; \\
\text { Exposición (3) }\end{array}$ \\
\hline
\end{tabular}




\begin{tabular}{|c|c|c|c|c|}
\hline $\begin{array}{l}\text { Autor, año } \\
\text { publicación, } \\
\text { referencia }\end{array}$ & $\begin{array}{l}\text { Población, } \\
\text { período de } \\
\text { estudio o } \\
\text { seguimiento }\end{array}$ & $\begin{array}{c}\text { Tamaño muestra y tipo de } \\
\text { estudio }\end{array}$ & Hallazgos principales & $\begin{array}{l}\text { Nivel de } \\
\text { calidad }\end{array}$ \\
\hline $\begin{array}{l}\text { Larsson et al., } \\
2005[24]\end{array}$ & $\begin{array}{l}\text { Suecia, solo } \\
\text { hombres, 1997- } \\
2004\end{array}$ & $\begin{array}{l}\text { Cohorte prospectiva } \\
(n=45550) \\
411 \text { casos incidentes de CCR. }\end{array}$ & $\begin{array}{l}\text { Hombres con DMT2 poseen } 49 \% \\
\text { de riesgo de padecer CCR (RR } \\
\text { ajustado: 1,45; IC95 \%: 1,11-1,89, RR } \\
\text { multivariado: 1,49; IC95 \%: 1,14- } \\
\text { 1,96). Regresión de Cox. } \\
\text { El riesgo observado se mantuvo en } \\
\text { análisis multivariado para cáncer de } \\
\text { colon (RR: 1,53; IC95 \%: 1,02-2,29) } \\
\text { y cáncer de recto (RR: 1,79; IC95 \%: } \\
1,18-2,73 \text { ) }\end{array}$ & $\begin{array}{l}\text { Bueno } \\
\text { Selección (4); } \\
\text { Comparabilidad } \\
\text { (1); } \\
\text { Desenlace (3) }\end{array}$ \\
\hline $\begin{array}{l}\text { Inoue et al., } 2006 \\
{[25]}\end{array}$ & $\begin{array}{l}\text { Japón, ambos } \\
\text { sexos, 1990- } \\
2003\end{array}$ & $\begin{array}{l}\text { Cohorte prospectiva } \\
(n=97771) \\
46548 \text { hombres: } 6,7 \% \text { DMT2 } \\
51223 \text { mujeres: } 3,1 \% \text { DMT2 }\end{array}$ & $\begin{array}{l}\text { En hombres con DMT2, se apreció } \\
\text { moderado incremento en el riesgo } \\
\text { de padecer cáncer de colon (HR: } \\
\text { 1,36; Ic95 \%: 1,00-1,85), mas no así } \\
\text { en mujeres. Regresión de Cox }\end{array}$ & $\begin{array}{l}\text { Bueno } \\
\text { Selección (4); } \\
\text { Comparabilidad } \\
(1) ; \\
\text { Desenlace (3) }\end{array}$ \\
\hline $\begin{array}{l}\text { Limburg et al., } \\
2006[26]\end{array}$ & $\begin{array}{l}\text { Estados } \\
\text { Unidos, ambos } \\
\text { sexos, 1970- } \\
1999\end{array}$ & $\begin{array}{l}\text { Cohorte retrospectiva } \\
(n=975) \\
51 \text { registros de CCR }\end{array}$ & 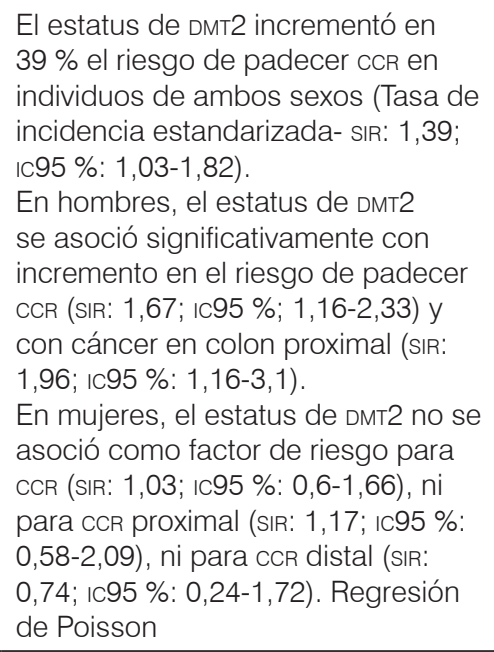 & $\begin{array}{l}\text { Bueno } \\
\text { Selección (4); } \\
\text { Comparabilidad } \\
\text { (1); } \\
\text { Desenlace (3) }\end{array}$ \\
\hline $\begin{array}{l}\text { Seow et al., } 2006 \\
{[27]}\end{array}$ & $\begin{array}{l}\text { China, ambos } \\
\text { sexos, 1993- } \\
1998\end{array}$ & $\begin{array}{l}\text { Cohorte prospectiva } \\
(n=63257) \\
636 \text { casos incidentes de ccR: } \\
352 \text { en hombres y } 284 \text { en } \\
\text { mujeres. }\end{array}$ & $\begin{array}{l}\text { El historial clínico de DMT2 se asoció } \\
\text { significativamente con el riesgo de } \\
\text { padecer CCR en hombres (RR: 1,5; } \\
\text { IC95 \%: 1,2-2,1) y mujeres (RR: 1,4; } \\
\text { IC95 \%: 1,0-1,9). } \\
\text { En análisis individuales por sitio } \\
\text { anatómico del cCR, el estatus de } \\
\text { DMT2 se asoció con el riesgo de } \\
\text { desarrollar cáncer en colon (RR: 1,5; } \\
\text { IC95 \%: 1,1-2,0) y cáncer de recto } \\
\text { (RR: 1,5; IC95\%: 1,0-2,1) en sexos } \\
\text { combinados. Regresión de Cox }\end{array}$ & $\begin{array}{l}\text { Bueno } \\
\text { Selección (4); } \\
\text { Comparabilidad } \\
\text { (1); } \\
\text { Desenlace (3) }\end{array}$ \\
\hline $\begin{array}{l}\text { Kuriki et al., } 2007 \\
\text { [28] }\end{array}$ & $\begin{array}{l}\text { Japón, ambos } \\
\text { sexos, 1988- } \\
2000\end{array}$ & $\begin{array}{l}\text { Casos y controles } \\
(n=59440) \\
762 \text { casos de ccR en hombres: } \\
427 \text { en colon y } 335 \text { en recto. } \\
549 \text { casos de ccR en mujeres: } \\
347 \text { en colon y } 202 \text { en recto. } \\
47768 \text { controles. }\end{array}$ & $\begin{array}{l}\text { En hombres adultos, se detectó } \\
\text { asociación significativa entre el } \\
\text { historial de DMT2 con el riesgo de } \\
\text { desarrollar CCR (OR: 2,19; ıc95 \%: } \\
\text { 1,56-3,07). } \\
\text { En mujeres adultas, se detectó } \\
\text { asociación significativa entre el } \\
\text { historial de DMT2 con el riesgo de } \\
\text { desarrollar CCR (OR: 2,40; Ic95\%: } \\
\text { 1,17-4,91). Regresión logística } \\
\text { unicondicional }\end{array}$ & $\begin{array}{l}\text { Bueno } \\
\text { Selección (4); } \\
\text { Comparabilidad } \\
\text { (1); } \\
\text { Exposición (3) }\end{array}$ \\
\hline
\end{tabular}




\begin{tabular}{|c|c|c|c|c|}
\hline $\begin{array}{l}\text { Autor, año } \\
\text { publicación, } \\
\text { referencia }\end{array}$ & $\begin{array}{l}\text { Población, } \\
\text { período de } \\
\text { estudio o } \\
\text { seguimiento }\end{array}$ & $\begin{array}{c}\text { Tamaño muestra y tipo de } \\
\text { estudio }\end{array}$ & Hallazgos principales & $\begin{array}{l}\text { Nivel de } \\
\text { calidad }\end{array}$ \\
\hline $\begin{array}{l}\text { Vinikoor et al., } \\
2009 \text { [29] }\end{array}$ & $\begin{array}{l}\text { Estados } \\
\text { Unidos, ambos } \\
\text { sexos, 1996- } \\
2006\end{array}$ & $\begin{array}{l}\text { Casos y controles } \\
(n=3752) \\
1688 \text { casos. } \\
2064 \text { controles. }\end{array}$ & $\begin{array}{l}\text { Individuos de tez blanca con } \\
\text { DMT2 exhibieron mayor riesgo de } \\
\text { desarrollar cáncer de colon (OR: 1,4, } \\
\text { Ic95 \%: 0,93-2,12) y cáncer de recto } \\
\text { (OR: 1,38, Ic95 \%: 1,0-1,9), que sus } \\
\text { contrapartes sin diabetes. } \\
\text { En individuos de tez blanca con } \\
\text { DMT2, el uso crónico con insulina se } \\
\text { asoció con el desarrollo de cáncer } \\
\text { de recto (OR: 1,74, Ic95 \%: 0,92- } \\
\text { 3,31). } \\
\text { En individuos afroamericanos, la } \\
\text { DMT2 no se asoció como factor de } \\
\text { riesgo para cáncer de colon (OR: } \\
\text { 1,17, Ic95 \%: 0,81-1,7), ni tampoco } \\
\text { para cáncer de recto (OR: 0,75, } \\
\text { IC95 \%: 0,44-1,28). Regresión } \\
\text { logística }\end{array}$ & $\begin{array}{l}\text { Bueno } \\
\text { Selección (4); } \\
\text { Comparabilidad } \\
\text { (1); } \\
\text { Exposición (3) }\end{array}$ \\
\hline $\begin{array}{l}\text { Ogunleye et al., } \\
2009 \text { [30] }\end{array}$ & $\begin{array}{l}\text { Escocia, ambos } \\
\text { sexos, 1993- } \\
2004\end{array}$ & $\begin{array}{l}\text { Cohorte prospectiva } \\
(n=28731) \\
9577 \text { con DMT2. } \\
19154 \text { sin DMT2. }\end{array}$ & $\begin{array}{l}\text { En individuos de ambos sexos, el } \\
\text { estatus de DMT2 se constituye en } \\
\text { un factor de riesgo para desarrollar } \\
\text { cáncer de colon (RR ajustado: 1,46; } \\
\text { Ic95 \%: 1,07-2,01), mas no para } \\
\text { cáncer de recto (RR: 0,53; Ic95 \%: } \\
0,25-1,1 \text { ). Regresión de Cox }\end{array}$ & $\begin{array}{l}\text { Bueno } \\
\text { Selección (4); } \\
\text { Comparabilidad } \\
\text { (1); } \\
\text { Desenlace (3) }\end{array}$ \\
\hline $\begin{array}{l}\text { Campbell et al., } \\
2010[31]\end{array}$ & $\begin{array}{l}\text { Estados } \\
\text { Unidos, ambos } \\
\text { sexos, 1992- } \\
2007\end{array}$ & $\begin{array}{l}\text { Cohorte prospectiva } \\
(n=84 \text { 194) } \\
73312 \text { hombres. } \\
81663 \text { mujeres. } \\
1567 \text { hombres y } 1242 \text { mujeres } \\
\text { desarrollaron ccR. }\end{array}$ & $\begin{array}{l}\text { En hombres, la DMT2 se asoció con } \\
\text { incremento en el riesgo de casos } \\
\text { incidentes de CCR (RR: 1,24; Ic95 \%: } \\
\text { 1,08-1,44) versus no diabéticos. } \\
\text { El riesgo de desarrollar CCR incidente } \\
\text { fue mayor en hombres con DMT2 } \\
\text { utilizando insulina (RR: 1,36; Ic95 \%: } \\
\text { 1,05-1,78), versus sus contrapartes } \\
\text { que no usaban insulina (RR: 1,22, } \\
\text { IC95\%: 1,04-1,45). } \\
\text { En mujeres, la DMT2 (RR: 1,01; } \\
\text { Ic95\%: 0,82-1,23) y el uso de } \\
\text { insulina (RR: 0,95; IC95 \%: 0,64-1,41) } \\
\text { no se asociaron con el riesgo de } \\
\text { padecer CCR incidente. Regresión } \\
\text { de Cox }\end{array}$ & $\begin{array}{l}\text { Bueno } \\
\text { Selección (4); } \\
\text { Comparabilidad } \\
\text { (1); } \\
\text { Desenlace (3) }\end{array}$ \\
\hline $\begin{array}{l}\text { Flood et al., } 2010 \\
\text { [32] }\end{array}$ & $\begin{array}{l}\text { Estados } \\
\text { Unidos, solo } \\
\text { mujeres, 1987- } \\
1998\end{array}$ & $\begin{array}{l}\text { Cohorte retrospectiva, } \\
\text { multicéntrico } \\
(n=45516) \\
41096 \text { mujeres: } 233 \text { casos } \\
\text { incidentes de ccR. }\end{array}$ & $\begin{array}{l}\text { Mujeres con DMT2 mostraron } \\
\text { elevado riesgo de desarrollar CCR (RR } \\
=1,60 \text {, IC95 \%: 1,18-2,18), versus } \\
\text { no diabéticas. } \\
\text { En mujeres con diagnóstico de } \\
\text { DMT2 entre los } 4 \text { y } 8 \text { años previos al } \\
\text { inicio del estudio, se observó mayor } \\
\text { riesgo de desarrollar CCR (RR: } 2,36 \text {; } \\
\text { IC95\%: 0,96-5,79). Regresión de } \\
\text { Cox }\end{array}$ & $\begin{array}{l}\text { Bueno } \\
\text { Selección (4); } \\
\text { Comparabilidad } \\
\text { (1); } \\
\text { Desenlace (3) }\end{array}$ \\
\hline
\end{tabular}




\begin{tabular}{|c|c|c|c|c|}
\hline $\begin{array}{l}\text { Autor, año } \\
\text { publicación, } \\
\text { referencia }\end{array}$ & $\begin{array}{l}\text { Población, } \\
\text { período de } \\
\text { estudio o } \\
\text { seguimiento }\end{array}$ & $\begin{array}{c}\text { Tamaño muestra y tipo de } \\
\text { estudio }\end{array}$ & Hallazgos principales & $\begin{array}{l}\text { Nivel de } \\
\text { calidad }\end{array}$ \\
\hline $\begin{array}{l}\text { He et al., } 2010 \\
\text { [33] }\end{array}$ & $\begin{array}{l}\text { Estados } \\
\text { Unidos, } \\
\text { multiétnico, } \\
\text { ambos sexos, } \\
\text { 1993-2006 }\end{array}$ & $\begin{array}{l}\text { Cohorte prospectiva } \\
(n=99143) \\
3549 \text { casos incidentes de CCR }\end{array}$ & 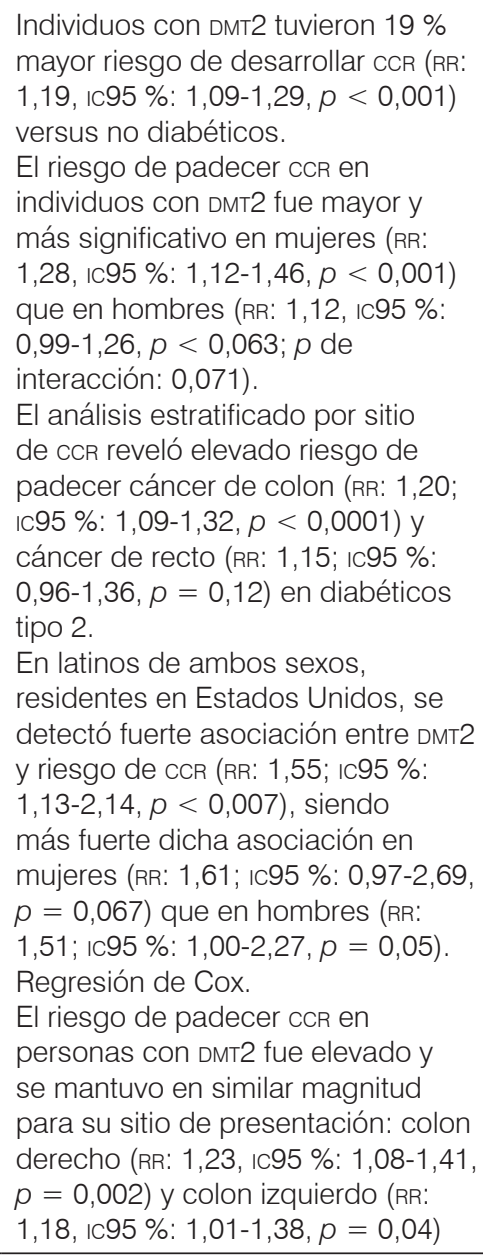 & $\begin{array}{l}\text { Bueno } \\
\text { Selección (4); } \\
\text { Comparabilidad } \\
(1) ; \\
\text { Desenlace (3) }\end{array}$ \\
\hline $\begin{array}{l}\text { Chodick et al., } \\
2010 \text { [34] }\end{array}$ & $\begin{array}{l}\text { Israel, ambos } \\
\text { sexos, 2000- } \\
2008\end{array}$ & $\begin{array}{l}\text { Cohorte retrospectiva } \\
(n=100 \text { 595) } \\
16721 \text { personas con DMT2 al } \\
\text { inicio de la cohorte. } \\
83874 \text { personas sin DMT2 al } \\
\text { inicio de la cohorte. }\end{array}$ & $\begin{array}{l}\text { En mujeres adultas, el estatus } \\
\text { de DMT2 se asoció con el riesgo } \\
\text { de padecer cáncer de colon (HR } \\
\text { ajustado: 1,52; ıc95 \%: 1,19-1,95), } \\
\text { mas no con cáncer de recto (HR } \\
\text { ajustado: 1,08; ıc95 \%: 0,69-1,69). } \\
\text { En hombres adultos, el estatus de } \\
\text { DMT2 no se asoció con el riesgo } \\
\text { de padecer cáncer de colon (HR } \\
\text { ajustado: 1,14; ıc95 \%: 0,91-1,44), } \\
\text { ni con cáncer de recto (HR ajustado: } \\
1,1 \text {; Ic95 \%: 0,74-1,61). Regresión } \\
\text { de Cox }\end{array}$ & $\begin{array}{l}\text { Bueno } \\
\text { Selección (4); } \\
\text { Comparabilidad } \\
\text { (1); } \\
\text { Desenlace (3) }\end{array}$ \\
\hline $\begin{array}{l}\text { Atchison et al., } \\
2011[35]\end{array}$ & $\begin{array}{l}\text { Estados } \\
\text { Unidos, solo } \\
\text { hombres, 1969- } \\
1996 \text {, }\end{array}$ & $\begin{array}{l}\text { Cohorte prospectiva } \\
(n=4501578) \\
584915 \text { diabéticos tipo } 2 . \\
3906763 \text { no diabéticos. }\end{array}$ & $\begin{array}{l}\text { Hombres adultos con DMT2 } \\
\text { exhibieron elevado riesgo de } \\
\text { desarrollar cáncer de colon (RR } \\
\text { ajustado: 1,20, IC95 \%: 1,16-1,25) y } \\
\text { cáncer de recto (RR ajustado: 1,20, } \\
\text { Ic95\%: 1,07-1,18). Regresión de } \\
\text { Poisson }\end{array}$ & $\begin{array}{l}\text { Bueno } \\
\text { Selección (4); } \\
\text { Comparabilidad } \\
\text { (1); } \\
\text { Desenlace (3) }\end{array}$ \\
\hline
\end{tabular}

10 | Universidad de Antioquia 


\begin{tabular}{|c|c|c|c|c|}
\hline $\begin{array}{l}\text { Autor, año } \\
\text { publicación, } \\
\text { referencia }\end{array}$ & $\begin{array}{l}\text { Población, } \\
\text { período de } \\
\text { estudio o } \\
\text { seguimiento }\end{array}$ & $\begin{array}{c}\text { Tamaño muestra y tipo de } \\
\text { estudio }\end{array}$ & Hallazgos principales & $\begin{array}{l}\text { Nivel de } \\
\text { calidad }\end{array}$ \\
\hline $\begin{array}{l}\text { Jarvandi et al., } \\
2013[36]\end{array}$ & $\begin{array}{l}\text { Estados } \\
\text { Unidos, ambos } \\
\text { sexos, 1996- } \\
2006\end{array}$ & $\begin{array}{l}\text { Cohorte prospectiva } \\
(n=484 \text { 020) } \\
7598 \text { casos incidentes de ccR: } \\
5130 \text { en hombres y } 2486 \text { en } \\
\text { mujeres. }\end{array}$ & 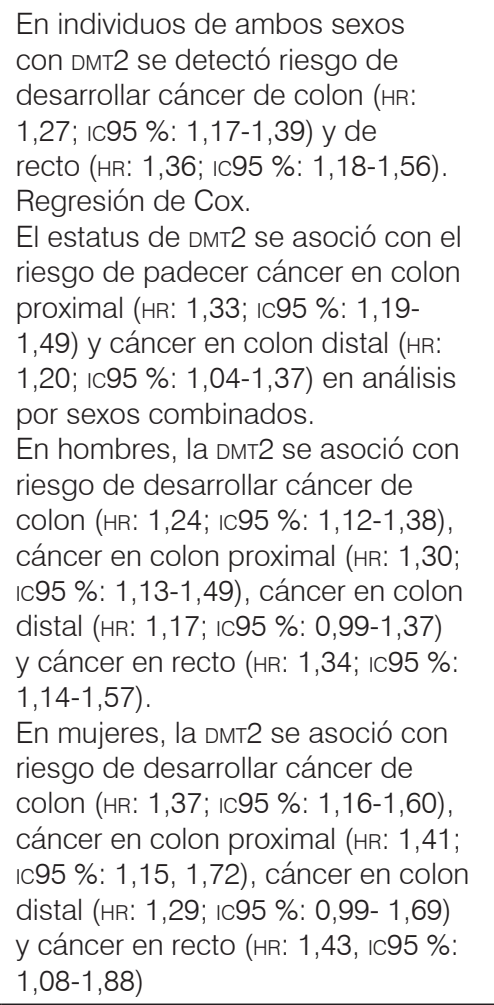 & $\begin{array}{l}\text { Bueno } \\
\text { Selección (4); } \\
\text { Comparabilidad } \\
(1) ; \\
\text { Desenlace (3) }\end{array}$ \\
\hline $\begin{array}{l}\text { Peeters et al., } \\
2015 \text { [37] }\end{array}$ & $\begin{array}{l}\text { Reino Unido, } \\
\text { ambos sexos, } \\
\text { 1987-2012 }\end{array}$ & $\begin{array}{l}\text { Cohorte retrospectiva } \\
(n=270785 \text { diabéticos tipo } \\
2) \\
2759 \text { registros de ccR en } \\
\text { diabéticos tipo } 2 \text {. } \\
1941 \text { registros de cáncer de } \\
\text { colon. } \\
819 \text { registros de cáncer de } \\
\text { recto. }\end{array}$ & $\begin{array}{l}\text { La DMT2 se asoció con un } \\
\text { incremento de } 1,3 \text { veces en el riesgo } \\
\text { de padecer CCR (HR: } 1,26 \text {, IC95 \%: } \\
1,18-1,33 \text { ) en sexos combinados. } \\
\text { Existe riesgo de padecer CCR } \\
\text { en individuos diabéticos tipo } 2 \\
\text { padeciendo obesidad durante } 4 \text { a } \\
8 \text { años (HR: } 1,19 \text {, Ic95 \%: 1,06-1,34) } \\
\text { y en diabéticos tipo } 2 \text { con más de } \\
8 \text { años de duración de la obesidad } \\
\text { (HR:1,28, IC95 \%: } 1,11-1,49 \text { ). } \\
\text { Regresión de Cox }\end{array}$ & $\begin{array}{l}\text { Bueno } \\
\text { Selección (4); } \\
\text { Comparabilidad } \\
\text { (1); } \\
\text { Desenlace (3) }\end{array}$ \\
\hline $\begin{array}{l}\text { Díaz et al., } 2015 \\
\text { [38] }\end{array}$ & $\begin{array}{l}\text { Puerto Rico, } \\
\text { ambos sexos, } \\
2005-2009\end{array}$ & $\begin{array}{l}\text { Casos y controles } \\
(n=451) \\
295 \text { casos } \\
156 \text { controles }\end{array}$ & $\begin{array}{l}\text { Mujeres con DMT2 exhiben casi tres } \\
\text { veces mayor riesgo de desarrollar } \\
\text { cCR O adenomas colorrectales (OR } \\
\text { ajustado: 2,74, Ic95 \%: 0,94-7,99) } \\
\text { que sus contrapartes sin diabetes y, } \\
\text { cerca de } 5 \text { veces mayor riesgo de } \\
\text { desarrollar ccR o adenomas en colon } \\
\text { proximal (oR ajustado: 4,83, Ic95 \%: } \\
\text { 1,25-18,58) comparado a controles. } \\
\text { Mujeres con DMT2 presentan } \\
\text { mayor riesgo de desarrollar ccR en } \\
\text { colon proximal (OR ajustado: } 7,19, \\
\text { Ic95 \%:1,44-35,96) que mujeres sin } \\
\text { diabetes. Regresión logística. } \\
\text { No fue observada ninguna } \\
\text { asociación entre DMT2 y riesgo/ } \\
\text { localización del ccR/adenomas en } \\
\text { hombres }\end{array}$ & $\begin{array}{l}\text { Bueno } \\
\text { Selección (4); } \\
\text { Comparabilidad } \\
(1) ; \\
\text { Exposición (3) }\end{array}$ \\
\hline
\end{tabular}




\begin{tabular}{|c|c|c|c|c|}
\hline $\begin{array}{c}\text { Autor, año } \\
\text { publicación, } \\
\text { referencia }\end{array}$ & $\begin{array}{l}\text { Población, } \\
\text { período de } \\
\text { estudio o } \\
\text { seguimiento }\end{array}$ & $\begin{array}{c}\text { Tamaño muestra y tipo de } \\
\text { estudio }\end{array}$ & Hallazgos principales & $\begin{array}{l}\text { Nivel de } \\
\text { calidad }\end{array}$ \\
\hline $\begin{array}{l}\text { Kort et al., } 2016 \\
{[39]}\end{array}$ & $\begin{array}{l}\text { Holanda, } \\
\text { ambos sexos, } \\
\text { 1986-2006 }\end{array}$ & $\begin{array}{l}\text { Cohorte prospectiva y casos- } \\
\text { cohorte } \\
(n=20 \text { 852) } \\
\text { Seguimiento de la cohorte } \\
\text { completa: } 20,3 \text { años } \\
\text { Seguimiento de los casos- } \\
\text { cohorte: } 17,3 \text { años }\end{array}$ & $\begin{array}{l}\text { Detectan asociación significativa } \\
\text { entre DMT2 y riesgo de desarrollar } \\
\text { cáncer en colon proximal en } \\
\text { mujeres (HR: 1,82, Ic95 \%: 1,11- } \\
\text { 2,98), mas no en hombres (HR: 1,37, } \\
\text { Ic95\%: 0,77-2,42), ni tampoco con } \\
\text { cáncer en colon distal y recto en } \\
\text { ambos sexos, en el grupo de casos- } \\
\text { cohorte. } \\
\text { En el enfoque de cohorte completa } \\
\text { (4593 casos de ccR y } 4292 \text { casos } \\
\text { de DMT2), encuentran } 82 \% \text { de } \\
\text { incremento en el riesgo de padecer } \\
\text { cáncer en colon proximal en } \\
\text { mujeres con DMT2 (HR ajustado: 1,44, } \\
\text { Ic95 \%:1,05-1,99) versus mujeres } \\
\text { sin DMT2. } \\
\text { Detectan incremento del riesgo } \\
\text { de aparición de cáncer en colon } \\
\text { proximal en mujeres con duración } \\
\text { de la DMT2 entre 0-10 años y } \\
\text { diagnóstico de DMT2 luego de los } \\
50 \text { años de edad (HR: } 1,89, \text { Ic95 \%: } \\
1,32-2,69 \text { ) y con larga duración de } \\
\text { la DMT2 y diagnóstico de DMT2 antes } \\
\text { de los } 50 \text { años de edad (HR: } 1,64 \text {, } \\
\text { Ic95 \%:1,17-2,32). Regresión de Cox }\end{array}$ & $\begin{array}{l}\text { Bueno } \\
\text { Selección (4); } \\
\text { Comparabilidad } \\
(1) ; \\
\text { Desenlace (3) }\end{array}$ \\
\hline $\begin{array}{l}\text { Rosato et al., } \\
2016[40]\end{array}$ & $\begin{array}{l}\text { Italia y España, } \\
\text { ambos sexos, } \\
2007-2013\end{array}$ & $\begin{array}{l}\text { Casos y controles } \\
(n=2741) \\
1147 \text { casos de cCR: } 14 \% \text { con } \\
\text { DMT2. } \\
1594 \text { controles: } 12 \% \\
\text { con DMT2. }\end{array}$ & $\begin{array}{l}\text { Individuos con DMT2 de ambos } \\
\text { países poseen riesgo significativo } \\
\text { de padecer cáncer de colon } \\
\text { (Riesgo: } 20 \% \text {, OR: 1,15, IC95 \%:0,89- } \\
\text { 1,55), cáncer en colon proximal (OR: } \\
\text { 1,53, IC95 \%:1,06-2,19), cáncer en } \\
\text { colon distal (OR: 0,94, IC95 \%: 0,66- } \\
\text { 1,36) y cáncer de recto (OR: 1,32, } \\
\text { Ic95\%: 0,94-1,87). } \\
\text { El uso crónico de insulina se asoció } \\
\text { con incremento en el riesgo de } \\
\text { cCR (OR: 2,2, Ic95 \%: 1,12-4,33), } \\
\text { fortaleciéndose este riesgo con } \\
\text { mayor duración en el uso de la } \\
\text { misma (OR: 8,18, Ic95 \%: } 2,06- \\
\text { 32,5, durante 10 o más años, } p \\
\text { Trend = 0,002). Regresión logística }\end{array}$ & $\begin{array}{l}\text { Bueno } \\
\text { Selección (4); } \\
\text { Comparabilidad } \\
(1) ; \\
\text { Exposición (3) }\end{array}$ \\
\hline $\begin{array}{l}\text { Dankner et al., } \\
2016 \text { [41] }\end{array}$ & $\begin{array}{l}\text { Israel, ambos } \\
\text { sexos, 2002- } \\
2012\end{array}$ & $\begin{array}{l}\text { Cohorte prospectiva } \\
(n=2186 \text { 196) } \\
567347 \text { individuos con DMT2. } \\
1618849 \text { individuos sin } \\
\text { diabetes. }\end{array}$ & $\begin{array}{l}\text { Detectan fuerte asociación entre } \\
\text { DMT2 incidente con incremento en } \\
\text { el riesgo -mayormente en mujeres- } \\
\text { de padecer CCR (HR:1,44, IC95 \%: } \\
\text { 1,33-1,55), como también, detectan } \\
\text { riesgo entre DMT2 prevalente y riesgo } \\
\text { de padecer ccR en ambos sexos (HR: } \\
\text { 1,48, IC95\%: 1,39-1,57). Regresión } \\
\text { de Cox }\end{array}$ & $\begin{array}{l}\text { Bueno } \\
\text { Selección (4); } \\
\text { Comparabilidad } \\
\text { (1); } \\
\text { Desenlace (3) }\end{array}$ \\
\hline
\end{tabular}




\begin{tabular}{|c|c|c|c|c|}
\hline $\begin{array}{l}\text { Autor, año } \\
\text { publicación, } \\
\text { referencia }\end{array}$ & $\begin{array}{l}\text { Población, } \\
\text { período de } \\
\text { estudio o } \\
\text { seguimiento } \\
\end{array}$ & $\begin{array}{c}\text { Tamaño muestra y tipo de } \\
\text { estudio }\end{array}$ & Hallazgos principales & $\begin{array}{l}\text { Nivel de } \\
\text { calidad }\end{array}$ \\
\hline $\begin{array}{l}\text { Kort et al., } 2017 \\
{[42]}\end{array}$ & $\begin{array}{l}\text { Holanda, } \\
\text { ambos sexos, } \\
\text { 1998-2010 }\end{array}$ & $\begin{array}{l}\text { Cohorte prospectiva } \\
(n=366770) \\
41716 \text { individuos con DMT2: } \\
394 \text { casos de cCR. } \\
325054 \text { no diabéticos: } 1939 \\
\text { casos de cCR. }\end{array}$ & $\begin{array}{l}\text { El estatus de DMT2 en adultos } \\
\text { de ambos sexos se asoció } \\
\text { significativamente con el riesgo } \\
\text { de padecer CCR (HR ajustado: 1,3; } \\
\text { Ic95 \%: 1,2-1,5. HR no ajustado: } \\
\text { 1,4; Ic95 \%: 1,3-1,6) y con riesgo } \\
\text { de cáncer en colon proximal (HR } \\
\text { ajustado: 1,7; Ic95 \%: 1,4-2,0). } \\
\text { En hombres, la DMT2 se asoció } \\
\text { con riesgo de desarrollar cCR (HR } \\
\text { ajustado: 1,3; Ic95 \%:1,1-1,5) y } \\
\text { cáncer en colon proximal (HR: } \\
\text { 1,6; Ic95 \%: 1,2-2,1). Este riesgo } \\
\text { incrementó en hombres menores de } \\
55 \text { años con DMT2 (HR: 2,0; Ic95 \%: } \\
\text { 1,0-3,8). } \\
\text { En mujeres, la DMT2 se asoció con } \\
\text { el riesgo de cCR (HR ajustado: 1,3; } \\
\text { Ic95 \%: 1,1-1,6) y cáncer en colon } \\
\text { proximal (HR ajustado: 1,8; Ic95 \%: } \\
\text { 1,4-2,3). Regresión de Cox }\end{array}$ & $\begin{array}{l}\text { Bueno } \\
\text { Selección (4); } \\
\text { Comparabilidad } \\
(1) ; \\
\text { Desenlace (3) }\end{array}$ \\
\hline $\begin{array}{l}\text { Sinagra et al., } \\
2017[43]\end{array}$ & $\begin{array}{l}\text { Italia, ambos } \\
\text { sexos, 2000- } \\
2008\end{array}$ & $\begin{array}{l}\text { Cohorte retrospectiva } \\
(n=741)\end{array}$ & $\begin{array}{l}\text { La duración de la DMT2 se asoció } \\
\text { con el riesgo de padecer cáncer } \\
\text { colorectal (HR: 1,93; Ic95 \%: 0,25- } \\
\text { 14,77, p < 0,001) en ambos sexos } \\
\text { combinados. Regresión logística } \\
\text { multivariada }\end{array}$ & $\begin{array}{l}\text { Bueno } \\
\text { Selección (4); } \\
\text { Comparabilidad } \\
\text { (1); } \\
\text { Desenlace (3) }\end{array}$ \\
\hline $\begin{array}{l}\text { Pang et al., } 2018 \\
\text { [44] }\end{array}$ & $\begin{array}{l}\text { China, ambos } \\
\text { sexos, 2004- } \\
2008\end{array}$ & $\begin{array}{l}\text { Cohorte prospectiva } \\
(n=512713) \\
3024 \text { casos incidentes de ccr: } \\
1745 \text { en colon y } 1716 \text { en recto. }\end{array}$ & $\begin{array}{l}\text { Adultos de ambos sexos con DMT2 } \\
\text { exhibieron riesgo de padecer CCR (HR } \\
\text { ajustado: 1,14; IC95 \%: 1,01-1,29), } \\
\text { cáncer de colon (HR ajustado: 1,14; } \\
\text { Ic95 \%: 0,97-1,34) y cáncer de recto } \\
\text { (HR ajustado: 1,11; Ic95 \%: 0,94- } \\
\text { 1,34). Regresión de Cox }\end{array}$ & $\begin{array}{l}\text { Bueno } \\
\text { Selección (4); } \\
\text { Comparabilidad } \\
\text { (1); } \\
\text { Desenlace (3) }\end{array}$ \\
\hline $\begin{array}{l}\text { Yanan et al., } \\
2018[45]\end{array}$ & $\begin{array}{l}\text { Estados } \\
\text { Unidos, ambos } \\
\text { sexos, 1980- } \\
2012\end{array}$ & $\begin{array}{l}\text { Cohorte retrospectiva } \\
(n=34763) \\
1631 \text { mujeres con DMT2. } \\
1129 \text { hombres con DMT2. }\end{array}$ & $\begin{array}{l}\text { En mujeres, el estatus de DMT2 se } \\
\text { asoció con el riesgo de padecer CCR } \\
\text { (HR ajustado: 1,32; Ic95 \%: 1,12- } \\
\text { 1,55). En forma similar, se detectó } \\
\text { asociación entre DMT2 y cCR durante } \\
\text { el período de seguimiento superior } \\
\text { a } 15 \text { años (HR ajustado: 1,37; Ic95 \%: } \\
\text { 1,0-1,87). } \\
\text { En hombres, la DMT2 se asoció } \\
\text { con el riesgo de desarrollar CCR (HR } \\
\text { ajustado: 1,57; ıc95 \%: 1,24-1,98). } \\
\text { Igualmente, se detectó asociación } \\
\text { entre DMT2 y cCR durante el período } \\
\text { de seguimiento de 0,1-5 años (HR } \\
\text { ajustado: 1,51; Ic95 \%: 1,08-2,12) y } \\
\text { de 5,1-10 años de seguimiento (HR } \\
\text { ajustado: 1,59; Ic95 \%: 1,06-2,38). } \\
\text { Regresión de Cox }\end{array}$ & $\begin{array}{l}\text { Bueno } \\
\text { Selección (4); } \\
\text { Comparabilidad } \\
\text { (1); } \\
\text { Desenlace (3) }\end{array}$ \\
\hline
\end{tabular}




\begin{tabular}{|c|c|c|c|c|}
\hline $\begin{array}{l}\text { Autor, año } \\
\text { publicación, } \\
\text { referencia }\end{array}$ & $\begin{array}{l}\text { Población, } \\
\text { período de } \\
\text { estudio o } \\
\text { seguimiento }\end{array}$ & $\begin{array}{c}\text { Tamaño muestra y tipo de } \\
\text { estudio }\end{array}$ & Hallazgos principales & $\begin{array}{l}\text { Nivel de } \\
\text { calidad }\end{array}$ \\
\hline $\begin{array}{l}\text { Overbeek et al., } \\
2019 \text { [46] }\end{array}$ & $\begin{array}{l}\text { Holanda, } \\
\text { ambos sexos, } \\
2006-2014\end{array}$ & $\begin{array}{l}\text { Cohorte retrospectiva } \\
(n=54 \text { 492) } \\
55055 \text { individuos con DMT2. } \\
99437 \text { individuos sin DMT2. }\end{array}$ & $\begin{array}{l}\text { Individuos de ambos sexos con } \\
\text { DMT2 tuvieron 1,3 veces mayor } \\
\text { riesgo de desarrollar ccR que sus } \\
\text { contrapartes sin diabetes. } \\
\text { Hombres con DMT2 exhibieron mayor } \\
\text { riesgo de desarrollar cáncer en } \\
\text { colon distal (HR: 1,42, Ic95 \%: 1,08- } \\
\text { 1,88) que mujeres con DMT2 (HR: } \\
\text { 0,86; Ic95 \%: 0,55-1,35). } \\
\text { Mujeres con DMT2 presentaron } \\
\text { mayor riesgo de padecer cáncer } \\
\text { en colon proximal (HR: 1,58; Ic95 \%: } \\
\text { 1,13-2,19) que hombres con } \\
\text { DMT2 (HR: 1,2; Ic95 \%: 0,87-1,65). } \\
\text { Regresión de Cox. } \\
\text { No fue observada asociación } \\
\text { estadísticamente significativa para } \\
\text { DMT2 y cáncer de recto en ambos } \\
\text { sexos }\end{array}$ & $\begin{array}{l}\text { Bueno } \\
\text { Selección (4); } \\
\text { Comparabilidad } \\
\text { (1); } \\
\text { Desenlace (3) }\end{array}$ \\
\hline $\begin{array}{l}\text { Miłek et al., } 2019 \\
\text { [47] }\end{array}$ & $\begin{array}{l}\text { Polonia, ambos } \\
\text { sexos, 2009- } \\
2011\end{array}$ & $\begin{array}{l}\text { Cohorte retrospectiva } \\
(n=976) \\
91 \text { con DMT2. } \\
885 \text { no diabéticos. }\end{array}$ & $\begin{array}{l}\text { Individuos con DMT2 no controlada } \\
\text { poseen mayor riesgo de desarrollar } \\
\text { CCR (OR: } 5,72 \text {, IC95 \%: 1,32-24,87) } \\
\text { que los no diabéticos. Regresión } \\
\text { logística. } \\
\text { Personas con DMT2 tuvieron mayor } \\
\text { incidencia de pólipos con cáncer } \\
\text { 9/91 (9,89\%), que individuos } \\
\text { no diabéticos 18/885 (2,03\%), } \\
\text { p < 0,001. } \\
\text { Los casos de ccR incidentes } \\
\text { ocurrieron con mayor frecuencia en } \\
\text { personas con DMT2 no controlada ( } p \\
=0,022) \text {. } \\
\text { Individuos con DMT2 tuvieron una } \\
\text { mayor incidencia de pólipos con } \\
\text { displasia de alto grado/carcinoma } \\
32 / 91 \text { (35,16 \%) en comparación a } \\
\text { no diabéticos } 136 / 885 \text { ( } 15,37 \% \text { ), } \\
p<0,001 \text {. Test Chi-cuadrado }\end{array}$ & $\begin{array}{l}\text { Bueno } \\
\text { Selección (4); } \\
\text { Comparabilidad } \\
\text { (1); } \\
\text { Desenlace (3) }\end{array}$ \\
\hline
\end{tabular}

CCR: Cáncer colorrectal; DMT2: Diabetes mellitus tipo 2; HR: Hazard ratio; IC: Intervalo de confianza; OR: Odds ratio; RR: Riesgo relativo; SIR: Tasa de incidencia estandarizada.

La evaluación de la calidad fue basada en la escala de Newcastle-Ottawa [15]. En la tabla 2, última columna, se muestra dicha evaluación.

\section{Resultados}

En la primera búsqueda resultaron 927515 documentos en inglés y 16051 en español, los cuales fueron añadidos al gestor bibliográfico EndNote y filtrados para comparar los duplicados. De esta forma, se obtuvieron 815632 publicaciones no duplicadas, entre las que se realizó una segunda búsqueda de estudios epidemiológicos que evaluaran el riesgo de desarrollar CCR en personas adultas con diagnóstico de DMT2 a nivel mundial. Esta segunda búsqueda arrojó 931 documentos, conteniendo 251 estudios de investigación original relacionados con la pregunta de investigación.

Posteriormente, se revisaron completamente uno a uno los estudios elegibles y aplicaron los criterios de inclusión y exclusión (Tabla 1). Finalmente, se incluyeron 32 investigaciones originales que describen a la DMT2 como factor de riesgo para el desarrollo del CCR a nivel mundial. Algunos aspectos sobre la normatividad en salud pública internacional y colombiana para el control de la diabetes tipo 2 y el cáncer colorrectal se comentan en la discusión. Los hallazgos de los estudios incluidos se presentan en la tabla 2. 


\section{Discusión}

Las enfermedades crónicas no transmisibles (ECNT), que incluyen al cáncer y la diabetes, son actualmente la principal causa de morbilidad, discapacidad, enfermedad prevenible y de muerte prematura en diversas regiones mundiales. Entre las ECNT, resaltan la DMT2 y el CCR, por exhibir marcada tendencia de incremento en sus proporciones de incidencia y mortalidad en las dos últimas décadas, y porque comparten factores de riesgo, comorbilidades y coexisten en millones de personas en diversas latitudes a nivel global. En este contexto, resultan preocupantes las proyecciones de la oms, la IARC y la International Diabetes Federation (IFD) sobre el aumento sustancial en la carga por enfermedad individual de CCR y DMT2 en los próximos años.

La presente revisión sistemática logró describir evidencias desde estudios epidemiológicos a nivel mundial, que reafirman a la DMT2 como un factor de riesgo importante para el desarrollo del CCR, y que permiten dar respuesta a nuestra pregunta de investigación, al señalar que individuos adultos de ambos sexos con DMT2 exhiben elevado y mayor riesgo de padecer cáncer de colon o de recto, comparado con individuos no diabéticos.

Es notable que el tamaño de las asociaciones de riesgo identificadas parecen ser dependientes del grupo étnico-geográfico estudiado, de la edad, el sexo y sitio/subsitio anatómico del CCR evaluado, así como del tiempo de evolución de la diabetes. Sin embargo, a pesar de las evidencias existentes a la fecha (2019) sobre la interrelación DMT2 y CCR, las intervenciones en prevención primaria y secundaria, y la tamización del cáncer de colon y recto en individuos diabéticos tipo 2 y en menores de 50 años solo se han implementado en pocos países.

En su conjunto, el anterior bloque de evidencias nos conducen a hipotetizar sobre la potencial existencia de variantes genéticas o epigenéticas en individuos con DMT2 en diversas etnias y geografías mundiales que, dependientes del estilo de vida, dieta y duración del tratamiento con insulina, pudieran conferir riesgo diferencial, sexo y sitio específico de padecer cáncer de colon y de recto, y que, a su vez, demarcarían el inicio y el grado de severidad clínica del CCR en diabéticos, como también modificarían el grado de respuesta al tratamiento quimio/radio-terapéutico e incluso quirúrgico, determinando subsecuentemente y de manera aditiva, los desenlaces sobre el pronóstico clínico y los indicadores de incidencia, prevalencia, mortalidad y carga asociada de ambas patologías $[55,56]$.

\section{Panorama internacional y colombiano sobre el control del CCR y DMT2}

Las normativas en salud pública internacionales y colombiana para poner en marcha medidas concertadas de lucha contra la diabetes, el cáncer y otras ECNT

Tabla 3. Normatividad en salud pública internacional y colombiana para el control de la DMT2 y CCR

\begin{tabular}{|c|c|c|c|}
\hline Título de la norma & Objetivos & Período & Referencia \\
\hline $\begin{array}{l}\text { Declaración política de la reunión de alto } \\
\text { nivel de la Asamblea General sobre la } \\
\text { prevención y el control de las enfermedades } \\
\text { no transmisibles. }\end{array}$ & $\begin{array}{l}\text { Examinar las medidas de prevención y } \\
\text { control de las ECNT. } \\
\text { Reconocer las repercusiones sociales y } \\
\text { económicas de la carga conjunta de las } \\
\text { ECNT. } \\
\text { Dictar medidas para la prevención y el } \\
\text { control de las ECNT. }\end{array}$ & 2011 & {$[48]$} \\
\hline $\begin{array}{l}\text { Plan de acción mundial para la prevención } \\
\text { y el control de las enfermedades no } \\
\text { transmisibles 2013-2020 de la oms }\end{array}$ & $\begin{array}{l}\text { Reconocer las principales ECNT que } \\
\text { deben priorizarse en su abordaje para } \\
\text { prevención y control. } \\
\text { Prevenir y reducir la carga de morbilidad } \\
\text { y mortalidad por ECNT. }\end{array}$ & 2013-2020 & [49] \\
\hline $\begin{array}{l}\text { Plan de acción para la prevención y } \\
\text { el control de las enfermedades no } \\
\text { transmisibles en las Américas 2013-2019 } \\
\text { OPS }\end{array}$ & $\begin{array}{l}\text { Establecer líneas estratégicas de } \\
\text { intervención de las ECNT en la región de } \\
\text { las Américas. }\end{array}$ & 2013-2019 & {$[50]$} \\
\hline
\end{tabular}




\begin{tabular}{|c|c|c|c|}
\hline Título de la norma & Objetivos & Período & Referencia \\
\hline $\begin{array}{l}\text { La Agenda } 2030 \text { y los objetivos de } \\
\text { desarrollo sostenible. Una oportunidad para } \\
\text { América Latina y el Caribe. CEPAL. }\end{array}$ & $\begin{array}{l}\text { Establecer lineamientos para mejorar } \\
\text { la sostenibilidad económica, social y } \\
\text { ambiental de los Estados de América } \\
\text { Latina y el Caribe. } \\
\text { Analizar y formular los medios para } \\
\text { alcanzar los objetivos de desarrollo } \\
\text { sostenible de la onu. }\end{array}$ & 2016 & {$[51]$} \\
\hline $\begin{array}{l}\text { Informe WHA } 70.12 \text { de la Séptima Asamblea } \\
\text { de Salud Mundial. Cancer prevention and } \\
\text { control in the context of an integrated } \\
\text { approach. }\end{array}$ & $\begin{array}{l}\text { Reconocer al cáncer como problema } \\
\text { prioritario de salud pública mundial. } \\
\text { Brindar recomendaciones a los } \\
\text { gobiernos sobre la implementación de } \\
\text { planes para la prevención y control del } \\
\text { cáncer. }\end{array}$ & 2017 & [52] \\
\hline $\begin{array}{l}\text { Plan decenal de salud pública de Colombia, } \\
\text { 2012-2021 }\end{array}$ & $\begin{array}{l}\text { Reducir la inequidad en salud. } \\
\text { Proponer medidas para mejorar las } \\
\text { condiciones de vida, la situación en } \\
\text { salud y disminuir la carga de enfermedad } \\
\text { por las ECNT }\end{array}$ & $2012-2021$ & [53] \\
\hline $\begin{array}{l}\text { Plan decenal para el control del cáncer en } \\
\text { Colombia, 2012-2021 }\end{array}$ & $\begin{array}{l}\text { Describir la situación actual del cáncer } \\
\text { en Colombia. } \\
\text { Establecer el marco normativo y político, } \\
\text { así como las metas y acciones para la } \\
\text { prevención y el control del cáncer en } \\
\text { población colombiana. }\end{array}$ & 2012-2021 & [54] \\
\hline
\end{tabular}

CCR: Cáncer colorrectal; CEPAL: Comisión Económica para América Latina y el Caribe; DMT2: Diabetes mellitus tipo 2; ECNT: Enfermedades crónicas no transmisibles, oms: Organización Mundial de la Salud; ops: Organización Panamericana de la Salud; wHA: Asamblea de Salud Mundial; ONU: Organización de las Naciones Unidas.

(véase Tabla 3), convergen en sus objetivos de reducir la mortalidad prevenible asociadas a ECNT, así como disminuir al mínimo la exposición a factores de riesgo comunes, aumentar la exposición a factores protectores y mitigar la carga socio-económica de estas enfermedades.

El estudio de las acciones internacionales para promover e implementar la prevención primaria de las ECNT permite observar que tales medidas han sido ineficaces en reducir significativamente la carga global y regional de las ECNT, lo que posiblemente esté relacionado con el desconocimiento del grado de progreso y beneficios otorgados por la prevención de tales patologías por parte de los gobiernos mundiales.

Entre algunas de las debilidades identificadas en los planes de salud pública sobre ECNT, destacan: la falta de cumplimiento y ejecución; la escasa prioridad sobre las intervenciones preventivas — promoción de la saludy regulación, y la falta de profesionalización sobre las actividades normativas relacionadas con los planes en salud, así como el no cumplimiento de las metas e improvisación en los procesos institucionales, con el subsiguiente efecto sobre la calidad y la eficacia en la prestación de los servicios de salud.
Recientemente, Colombia fue incluido en el grupo principal de países con la mayor incidencia y mortalidad prematura por cánceres del mundo, abarcando el CCR [4]. En 2012, el Instituto Nacional de Cancerología de Colombia reconoció la existencia de una tendencia al alza en la mortalidad general por CCR en ambos sexos durante las últimas décadas [54]. Igualmente, el Observatorio Nacional del Cáncer de Colombia señaló que el CCR es uno de los cánceres que generan la mayor probabilidad de morir, y justificó como prioritario el investigar en profundidad las causas subyacentes de estos desenlaces [57].

Por consiguiente, el CCR fue incluido en el "Plan decenal para el control del cáncer en Colombia 20122021 " entre los cinco cánceres con mortalidad prematura evitable, y se planteó intervenirlo con actividades de atención primaria, secundaria y terciaria [54]. Estas actividades establecen metas enmarcadas en la línea estratégica de detección temprana, que incluyen: ampliar la cobertura de pruebas de tamización, incrementar la proporción de casos de cáncer detectados en estadios tempranos, así como garantizar el acceso oportuno y de calidad al diagnóstico y al tratamiento de pacientes positivos en las pruebas de tamización. 
En sustento de lo anterior, el Ministerio de Salud y Protección Social de Colombia, mediante la Guía de práctica clínica (GPC) para la detección temprana, diagnóstico, tratamiento integral, seguimiento y rehabilitación del cáncer de colon y recto [58], estableció que la estrategia óptima de tamización para CCR en población colombiana en riesgo consiste en practicar la prueba de sangre oculta en materia fecal inmunoquímica (SOMF) cada dos años y colonoscopia cuando exista un resultado positivo de SOMF o colonoscopia cada diez años - cuando esta se encuentre disponible - , entre los 50 y 75 años de edad.

Sin embargo, el sistema de salud colombiano carece de un programa de tamización organizado para la detección temprana y oportuna del CCR, pues existe solo de oportunidad. La guía anteriormente mencionada no brinda recomendaciones sobre el uso de biomarcadores bioquímicos, ni genético-moleculares para el tamizaje del riesgo poblacional, ni tampoco para el diagnóstico oportuno del CCR en sus estadios tempranos, en mayores de 50 años. De igual manera, esta guía no aborda la tamización en población menor de 50 años, sin antecedentes de riesgo familiar o personal - mucho menos en pacientes con DMT2 - , ni tampoco contempla la práctica de colonoscopia antes de los 10 años del primer resultado de colonoscopia negativa en mayores de 50 años. Lo anterior pudiera estar contribuyendo a un diagnóstico y tratamiento tardíos, así como al desfavorable pronóstico de los pacientes con $\mathrm{CCR}$ avanzado y al aumento exagerado de la carga de la enfermedad para el sistema de salud colombiano [59].

Adicionalmente, esta guía no reconoce a la DMT2 como factor de riesgo para el desarrollo, severidad y pronóstico del $\mathrm{CCR}$ en población colombiana, ni tampoco permite identificar subgrupos de individuos con CCR, en mayor o menor riesgo de padecer DMT2, que pudiera influir negativamente en los desenlaces de la enfermedad oncológica. Así mismo, el algoritmo actual de clasificación del riesgo en CCR no brinda claridad, pues no se aprecian los criterios para definir el tipo y el nivel de riesgo en bajo, medio y alto. Tampoco priorizan el tipo de intervención clínica según el nivel de riesgo, sino por antecedentes de riesgo heredofamiliar.

En relación con la diabetes, Colombia se ubica segundo entre los países suramericanos con el mayor número de personas afectadas por esta patología [60]. Y los reportes epidemiológicos nacionales ubican a la diabetes entre las cinco primeras causas de mortalidad general, y su prevalencia es mayor en individuos de áreas urbanas que rurales, afectando en mayor número a mujeres. Según edad de los afectados, el $88 \%$ de los casos de diabetes se presentan en personas mayores de 45 años de edad [61]. Sin embargo, el perfil de diabetes de Colombia, elaborado por la oms en 2016, reveló una tendencia de incremento progresivo de la enfermedad en mayores de 30 años de ambos sexos [62]. Empero, en el país no existe cobertura total en la prestación de servicios diagnósticos y de tratamiento para la diabetes. Se estima que cerca del $50 \%$ de los diabéticos aún no han sido diagnosticados [61], mientras que el $21 \%$ de los que reciben tratamiento para diabetes no alcanzan objetivos terapéuticos [63].

En forma similar, Colombia no cuenta con un sistema de registro nacional de diabetes y existe escaso número de estudios de corte trasversal y cohorte prospectivo sobre diabetes. Los estudios existentes han sido conducidos en áreas poblaciones urbanas y rurales que incluyen pequeño tamaño muestral, lo cual no permite estimar con precisión o exactitud, y comparar estadísticamente la prevalencia, incidencia y mortalidad general, o estratificar por grupos étnicos o geográficos la presentación real de la enfermedad al nivel nacional. Por tanto, es altamente probable que las proporciones actuales de prevalencia, incidencia y mortalidad para diabetes en Colombia se encuentren subestimadas.

Por su parte, al analizar la Guía de práctica clínica para el diagnóstico, tratamiento y seguimiento de la diabetes mellitus tipo 2 en población mayor de 18 años, en Colombia, del Ministerio de Salud y Protección Social [64], observamos que la misma no clasifica a la DMT2 como un factor de riesgo para el desarrollo, la severidad y el pronóstico del CCR, y viceversa, ni tampoco como una complicación de cualquier tipo de cáncer. Así mismo, no vincula el uso de medicamentos hipoglicemiantes orales o sistémicos para el tratamiento de la DMT2 con el desarrollo, severidad y pronóstico del CCR. Aún más, el sistema de salud estatal no reconoce el grado de interrelación epidemiológica y biológica entre ambas patologías. De igual modo, ninguna de las mencionadas guías considera la potencial existencia de subgrupos de personas con DMT2 en riesgo diferencial de padecer CCR y otros cánceres en población colombiana.

\section{Recomendaciones y perspectivas futuras}

El CCR, de acuerdo con la tendencia observada en Latinoamérica, se ubicará muy pronto en el segundo lugar de incidencia y mortalidad en el sexo femenino, favorecido por el desarrollo social y económico de la región, los riesgos hormonales-reproductivos y los hábitos dietarios que conducen a la obesidad y su continuo metabólico, la diabetes mellitus tipo 2.

En Colombia, es necesario adelantar estudios multicéntricos de cohorte prospectivo y de corte trasversal, que incluyan muestras poblacionales más grandes y heterogéneas, tanto de áreas urbanas y rurales a nivel nacional, para dilucidar el panorama epidemiológico de la diabetes y con ello tomar medidas concertadas en salud pública, para lograr su control eficaz. Además, es urgente la creación del registro único 
de diabetes de Colombia, lo cual permitirá identificar los grupos poblaciones y las geografías con mayores necesidades de intervención.

Es claro que la pandemia de obesidad, vinculada con el CCR, ya sea directamente o con el paso previo por la diabetes mellitus, se debe abordar con políticas de salud pública. Las intervenciones deben orientarse a materializar las acciones que están propuestas en el "Plan decenal del control del cáncer 2012-2021” que priorizó el CCR [54]. Además, deben incluirse, en la prevención secundaria, la tamización con sangre oculta por prueba inmunoquímica y la realización de colonoscopia, de ser procedente cada dos años, a todos los pacientes mayores de 25 años con diagnóstico de diabetes mellitus tipo 2 de mínimo cinco años de evolución.

Igualmente, sería conveniente implementar, en Colombia, programas de prevención de factores de riesgo para ECNT, con mayor énfasis en diabetes y cáncer, acompañados de un programa más agresivo para la detección temprana del CCR en pacientes menores de 50 años — sin riesgo heredofamiliar aparente-, en especial en el grupo con DMT2 y en aquellos que reciben tratamiento crónico con insulina, que integre la realización de marcadores bioquímicos y genéticomoleculares útiles en la detección de los estadios precursores e iniciales del CCR.

Otra contingencia que ayudaría a un mayor entendimiento de la asociación entre DMT2 y CCR es la realización de investigación traslacional en las diferentes poblaciones de Colombia, que revele interacciones entre factores ambientales y de comportamiento, medicamentos y polimorfismos genéticos funcionales para la etiología del primer paso tumoral, es decir, los adenomas en colon y recto. Por otro lado, también es conveniente ahondar en la conducción de estudios prospectivos sobre DMT2 y CCR en población colombiana, si consideramos que la comorbilidad de DMT2 empobrece los desenlaces y la supervivencia global del paciente con CCR.

En este sentido, es menos claro si la DMT2 en individuos con $\mathrm{CCR}$ es expresión del tratamiento farmacológico o es una complicación per se del CCR. Por tanto, es necesario conducir estudios de epidemiología molecular y básicos experimentales, tendientes a dilucidar los efectos biológico-moleculares de la insulina y de otros grupos de hipoglicemiantes en el desarrollo del CCR en individuos con DMT2. Además, merece nuestra atención el estudio del papel de las hormonas sexuales en el desarrollo del CCR en personas con DMT2.

\section{Conflicto de interés}

Ninguno declarado por los autores.

\section{Financiación}

El presente manuscrito contó con el apoyo de las bases de datos bibliográficas de la Universidad de Cartagena y la Universidad de Sucre.

\section{Declaración de responsabilidad}

Los puntos de vista declarados en el contenido del presente manuscrito son originales y responsabilidades del autor principal y los coautores, más no de las instituciones en las que laboran los autores.

\section{Declaración de autoría}

Deivis Javier Villanueva-Pájaro: Es el autor principal y de correspondencia del presente artículo, por cuanto planificó el diseño metodológico, la construcción, la conducción del documento y la ejecución de las actividades tendientes a cumplir con las exigencias de elaboración y aprobación final de la presente revisión sistemática. Además, participó de primera línea en la búsqueda masiva, estructurada, sistemática, comparativa y de clasificación de la bibliografía científica pertinente a ser incluida. Igualmente, participó en la redacción crítica del documento, como también sirvió de revisor e interlocutor crítico del trabajo, aportando al contenido intelectual en epidemiología, salud pública y metodología de la investigación científica y bibliográfica. Así mismo, fue el responsable de garantizar la integridad y la calidad de cada componente del documento, así como el encargado de unificar los criterios y aportes propios y de los coautores.

Edgar Ernesto Vergara-Dagobeth: Es coautor en el presente artículo, por cuanto participó en la revisión estructurada, sistemática y comparativa de las investigaciones originales pertinentes, ayudando al autor principal a clasificar e identificar los niveles de evidencia científicos, así como a calificar la calidad de la evidencia y a traducir los valores estadísticos en significados clínicos, epidemiológicos y de salud pública. Asimismo, contribuyó intelectualmente a la redacción crítica y metodológica del documento.

Amileth Suárez-Causado: Es coautora en el presente artículo, por cuanto brindó la idea de investigación, asesoró al autor principal en aspectos de metodología de la investigación científica y bibliográfica, participó en la revisión estructurada y sistemática de información, revisó los avances en la escritura del manuscrito y contribuyó a su corrección. Igualmente, ayudó en la redacción de la discusión.

Rubén Darío Gómez-Arias: Es coautor en el presente artículo, por cuanto participó activamente en la selección y revisión de las políticas en salud pública 
para el control de la diabetes y el cáncer colorrectal a nivel mundial y nacional. También propuso la inclusión del marco normativo en salud pública para el control de las enfermedades crónicas en general y de la diabetes y el cáncer colorrectal en particular. Además, aportó a la construcción intelectual y crítica de los aspectos epidemiológicos y en salud pública del artículo. Finalmente, integró componentes epistemológicos, humanistas y de filosofía de la ciencia, para dar claridad al autor principal y coautores, sobre la interpretación y significados de los hallazgos, y contribuyó a la crítica en la discusión del artículo.

\section{Consideraciones éticas}

El presente manuscrito no viola las normas éticas internacionales en materia de publicación, por parte de los autores y los coautores, ni tampoco compromete a las instituciones de filiación de los autores.

\section{Referencias}

1. Bray F, Ferlay J, Soerjomataram I, et al. Global cancer statistics 2018: GLOBOCAN estimates of incidence and mortality worldwide for 36 cancers in 185 countries. CA Cancer J Clin. 2018;68(6):394-424. DOI: https://doi.org/10.3322/caac.21492

2. Fitzmaurice C, Allen C, Barber RM, et al. Global, regional, and national cancer incidence, mortality, years of life lost, years lived with disability, and disability-adjusted life-years for 32 cancer groups, 1990 to 2015: A systematic analysis for the Global Burden of Disease study. JAMA Oncol. 2017;3(4):524-48. DOI: https:// doi.org/10.1001/jamaoncol.2016.5688

3. Ferlay J, Soerjomataram I, Dikshit R, et al. Cancer incidence and mortality worldwide: Sources, methods and major patterns in GLOBOCAN 2012. Int J Cancer. 2015;136(5):E359-86. DOI: https://doi.org/10.1002/ijc.29210

4. Arnold M, Sierra MS, Laversanne M, et al. Global patterns and trends in colorectal cancer incidence and mortality. Gut. 2017;66(4):683-91. DOI: http://dx.doi.org/10.1136/ gutjnl-2015-310912

5. Sadeghi H, Majid S, Aghajani M, et al. Colorectal cancer in the world: Incidence, mortality and risk factors. Biomed Res Ther 2017;4(10):1656-75. DOI: http://dx.doi.org/10.15419/bmrat. $\mathrm{v} 4 \mathrm{i} 10.372$

6. Ferlay J, Colombet M, Soerjomataram I, et al. Estimating the global cancer incidence and mortality in 2018: GLOBOCAN sources and methods. Int J Cancer. 2019;144(8):1941-53. DOI: https://doi.org/10.1002/ijc.31937.

7. Sierra MS, Forman D. Burden of colorectal cancer in Central and South America. Cancer Epidemiol. 2016;44(Supl. 1):S74-S81. DOI: https://doi.org/10.1016/j.canep.2016.03.010

8. Ferreira EJ, Meléndez HJ. Características clínicas, demográficas e histopatológicas de los pacientes con cáncer colorrectal del Hospital Universitario de Santander. Rev Colomb Cir. [internet] 2012 [citado 2020 ene. 27]; 27:213-20. Disponible en: http:// www.scielo.org.co/pdf/rcci/v27n3/v27n3a6.pdf

9. Ezzati M, et al. Worldwide trends in diabetes since 1980: A pooled analysis of 751 population-based studies with 4.4 million participants. Lancet. 2016;387(10027):1513-30. DOI: https://doi. org/10.1016/S0140-6736(16)00618-8

10. International Diabetes Federation (IFD) [internet]. IFD Diabetes Atlas. 8.a ed. Bruselas: IFD [internet]; 2017. [citado 2019 sep. 21]. Disponible en: https://www.idf.org/e-library/epidemiologyresearch/diabetes-atlas/134-idf-diabetes-atlas-8th-edition.html

11. Jaacks LM, Siegel KR, Gujral UP, et al. Type 2 diabetes: A 21st century epidemic. Best Pract Res Clin Endocrinol Metab. 2016;30(3):331-43. DOI: https://doi.org/10.1016/j. beem.2016.05.003

12. Shaw JE, Sicree RA, Zimmet PZ. Global estimates of the prevalence of diabetes for 2010 and 2030. Diabetes Res Clin Pract. 2010;87(1):4-14. DOI: https://doi.org/10.1016/j. diabres.2009.10.007

13. Tamayo DC. Diabetes en Colombia. Construyendo el panorama desde la perspectiva del observatorio de diabetes de Colombia. Libro blanco del observatorio de diabetes de Colombia. 2013. Organización para la Excelencia de la Salud. [internet]; 2014 [citado 2019 sep. 21]. Disponible en: https://www.odc.org.co/ files/libro_blanco_2013.pdf

14. Moher D, Liberati A, Tetzlaff J, et al. Preferred reporting items for systematic reviews and meta-analyses: The PRISMA Statement. PLoS Med. 2009; 6(7): e1000097. DOI: https://doi.org/10.1371/ journal.pmed.1000097

15. Luchini C, Stubbs B, Solmi M, et al. Assessing the quality of studies in meta-analyses: Advantages and limitations of the Newcastle Ottawa Scale. World J Meta-Anal. 2017;5(4):80-84. DOI: http://dx.doi.org/10.13105/wjma.v5.i4.80

16. La Vecchia C, D'Avanzo B, Negri E, et al. History of selected diseases and the risk of colorectal cancer. Eur $\mathrm{J}$ Cancer. 1991;27(5):582-6. DOI: https://doi.org/10.1016/02775379(91)90223-Z

17. Steenland K, Nowlin S, Palu S. Cancer incidence in the national health and nutrition survey I. Follow-up data: Diabetes, cholesterol, pulse and physical activity. Cancer Epidemiol Biomarkers Prev. $1995 ; 4(8): 807-11$

18. La Vecchia C, Negri E, Decarli A, et al. Diabetes mellitus and colorectal cancer risk. Cancer Epidemiol Biomarkers Prev. 1997;6(12):1007-10

19. Le Marchand L, Wilkens LR, Kolonel LN, et al. Associations of sedentary lifestyle, obesity, smoking, alcohol use, and diabetes with the risk of colorectal cancer. Cancer Res. 1997;57(21):478794

20. Hu FB, Manson JE, Liu S, et al. Prospective study of adult onset diabetes mellitus (type 2) and risk of colorectal cancer in women. J Natl Cancer Inst. 1999;91(6):542-7. DOI: https://doi.org/10.1093/ jnci/91.6.542

21. Nilsen TI, Vatten LJ. Prospective study of colorectal cancer risk and physical activity, diabetes, blood glucose and BMI: Exploring the hyperinsulinaemia hypothesis. Br J Cancer. 2001;84(3):41722. DOI: https://doi.org/10.1054/bjoc.2000.1582

22. Limburg PJ, Anderson KE, Johnson TW, et al. Diabetes mellitus and subsite-specific colorectal cancer risks in the Iowa Women's Health Study. Cancer Epidemiol Biomarkers Prev. [internet]. 2005 [citado 2020 ene. 27];14(1):2277. Disponible en: https:// cebp.aacrjournals.org/content/14/9/2277.2.long

23. Yang YX, Hennessy S, Lewis JD. Type 2 diabetes mellitus and the risk of colorectal cancer. Clin Gastroenterol Hepatol. 2005;3(6):587-94. DOI: https://doi.org/10.1016/S15423565(05)00152-7 
24. Larsson SC, Giovannucci E, Wolk A. Diabetes and colorectal cancer incidence in the cohort of Swedish men. Diabetes Care. 2005;28(7):1805-07. DOI: https://doi.org/10.2337/ diacare.28.7.1805

25. Inoue M, Iwasaki M, Otani T, et al. Diabetes mellitus and the risk of cancer: Results from a large-scale population-based cohort study in Japan. Arch Intern Med. 2006;166(17):1871-7. DOI: https://doi.org/10.1001/archinte.166.17.1871

26. Limburg PJ, Vierkant RA, Fredericksen ZS, et al. Clinically confirmed type 2 diabetes mellitus and colorectal cancer risk: A population-based, retrospective cohort study. Am J Gastroenterol. 2006;101(8):1872-9

27. Seow A, Yuan JM, Koh WP, et al. Diabetes mellitus and risk of colorectal cancer in the Singapore Chinese Health Study. J Nat Cancer Inst. 2006;98(2):135-8. DOI: https://doi.org/10.1093/jnci/ djj015

28. Kuriki K, Hirose K, Tajima K. Diabetes and cancer risk for all and specific sites among Japanese men and women. Eur J Cancer Prev. 2007;16(1): 83-89. DOI: https://doi.org/10.1097/01. cej.0000228404.37858.40

29. Vinikoor LC, Long MD, Keku TO, et al. The association between diabetes, insulin use, and colorectal cancer among Whites and African Americans. Cancer Epidemiol Biomarkers Prev. 2009;18(4):1239-42. DOI: https://doi.org/10.1158/1055-9965. EPI-08-1031

30. Ogunleye AA, Ogston SA, Morris AD, et al. A cohort study of the risk of cancer associated with type 2 diabetes. $\mathrm{Br} \mathrm{J}$ Cancer. 2009;101(7):1199-201. DOI: https://doi.org/10.1038/ sj.bjc. 6605240

31. Campbell PT, Deka A, Jacobs EJ, et al. Prospective study reveals associations between colorectal cancer and type 2 diabetes mellitus or insulin use in men. Gastroenterology. 2010;139(4):1138-46. DOI: https://doi.org/10.1053/j.gastro.2010.06.072

32. Flood A, Strayer L, Schairer C, et al. Diabetes and risk of incident colorectal cancer in a prospective cohort of women. Cancer Causes Control. 2010;21(8):1277-84. DOI: https://doi. org/10.1007/s10552-010-9555-0

33. He J, Stram DO, Kolonel LN, et al. The association of diabetes with colorectal cancer risk: The multiethnic cohort. Br J Cancer. 2010;103(1):120-6. DOI: https://doi.org/10.1038/sj.bjc.6605721

34. Chodick G, Heymann AD, Rosenmann L, et al. Diabetes and risk of incident cancer: A large population-based cohort study in Israel. Cancer Causes Control. 2010;21(6):879-87. DOI: https:// doi.org/10.1007/s10552-010-9515-8

35. Atchison EA, Gridley G, Carreon JD, et al. Risk of cancer in a large cohort of U.S. veterans with diabetes. Int J Cancer. 2011;128(3):635-43. DOI: https://doi.org/10.1002/ijc.25362

36. Jarvandi S, Davidson NO, Schootman M. Increased risk of colorectal cancer in type 2 diabetes is independent of diet quality PloS One. 2013;8(9):e74616. DOI: https://doi.org/10.1371/ journal.pone.0074616

37. Peeters PJ, Bazelier MT, Leufkens H, et al. The risk of colorectal cancer in patients with type 2 diabetes: Associations with treatment stage and obesity. Diabetes Care. 2015;38(3):495-502. DOI: https://doi.org/10.2337/dc14-1175

38. Díaz-Algorri Y, Lozada ME, López SM, et al. Type 2 diabetes mellitus and colorectal neoplasia risk in Hispanics: A case-control study. J Diabetes Complications. 2015;29(4):502-7. DOI: https:// doi.org/10.1016/j.jdiacomp.2015.01.010

39. Kort S de, Simons CC, Brandt PA van den, et al. Diabetes mellitus type 2 and subsite-specific colorectal cancer risk in men and women: Results from the Netherlands Cohort Study on diet and cancer. Eur J Gastroenterol Hepatol. 2016; 28(8):896-903. DOI: https://doi.org/10.1097/MEG.0000000000000626

40. Rosato V, Tavani A, Gracia-Lavedan E, et al. Type 2 diabetes, antidiabetic medications, and colorectal cancer risk: two casecontrol studies from Italy and Spain. Front Oncol. 2016;6:1-7. DOI: https://doi.org/10.3389/fonc.2016.00210

41. Dankner R, Boffetta P, Balicer RD, et al. Time-dependent risk of cancer after a diabetes diagnosis in a cohort of 2.3 million adults. Am J Epidemiol. 2016;183(12):1098-106. DOI: https://doi. org/10.1093/aje/kwv290

42. Kort S de, Masclee AAM, Sanduleanu S, et al. Higher risk of colorectal cancer in patients with newly diagnosed diabetes mellitus before the age of colorectal cancer screening initiation. Sci Rep. 2017;7:1-8. DOI: https://doi.org/10.1038/srep46527

43. Sinagra E, Guarnotta V, Raimondo D, et al. Colorectal cancer in patients with type 2 diabetes mellitus: A single-center experience. J Biol Regul Homeost Agents. 2017;31(4):1101-7.

44. Pang Y, Kartsonaki C, Guo Y, et al. Diabetes, plasma glucose and incidence of colorectal cancer in Chinese adults: A prospective study of 0.5 million people. J Epidemiol Community Health. 2018;72(10):919-25. DOI: https://doi.org/10.1136/jech-2018210651

45. Ma Y, Yang W, Song M, et al. Type 2 diabetes and risk of colorectal cancer in two large U.S. prospective cohorts. $\mathrm{Br} \mathrm{J}$ Cancer. 2018;119(11):1436-42. DOI: https://doi.org/10.1038/ s41416-018-0314-4

46. Overbeek JA, Kuiper JG, Heijden A van der, et al. Sex- and sitespecific differences in colorectal cancer risk among people with type 2 diabetes. Int J Colorectal Disease. 2019;34(2):269-76. DOI: https://doi.org/10.1007/s00384-018-3191-7

47. Miłek T, Forysiński K, Myrcha P, et al. Diabetes association of polyps and colon cancer. Pol Przegl Chi. 2019;91(4):9-12. DOI: https://doi.org/10.5604/01.3001.0013.2588

48. Organización de la Naciones Unidas (ONU). Declaración política de la reunión de alto nivel de la Asamblea General sobre la prevención y el control de las enfermedades no transmisibles. Documento A/66/L.1 P1-14. New York: ONU [internet]; 2011 [citado 2019 oct. 15]. Disponible en: https:/undocs.org/es/ A/66/L.1

49. Organización Mundial de la Salud (OMS), Plan de acción mundial para la prevención y el control de las enfermedades no transmisibles 2013-2020. Ginebra: OMS [internet]; 2013 [citado 2019 oct. 15]. Disponible en: https://www.who.int/cardiovascular diseases/15032013_updated_revised_draft_action_plan_spanish. pdf?ua $=1$

50. Organización Panamericana de la Salud (OPS). Plan de acción para la prevención y el control de las enfermedades no transmisibles en las Américas 2013-2019. Washington: OPS [internet]; 2014 [citado 2019 oct. 15]. Disponible en: https://www.paho.org/hq/ dmdocuments/2014/NCD-SP-low.pdf

51. Comisión Económica para América Latina y el Caribe (CEPAL). Agenda 2030 y los objetivos de desarrollo sostenible. Una oportunidad para América Latina y el Caribe. Santiago de Chile: CEPAL [internet]; 2016 [citado 2019 oct. 15]; Disponible en: http://repositorio.cepal.org/bitstream/handle/11362/40155/24/ S1801141_es.pdf

52. World Health Organization (WHO). Seventieth World Health Assembly. Cancer prevention and control in the context of an integrated approach. Génova: WHO [internet]; 2017 [citado 2019 
oct. 15]. Disponible en: http://apps.who.int/gb/ebwha/pdf_files/ WHA70/A70 R12-en.pdf

53. Colombia, Ministerio de Salud y Protección Social (MSPS). Plan decenal de salud pública, PDSP, 2012-2021. Bogotá: MSPS [internet]; 2013 [citado 2019 oct. 15]. Disponible en: https://www. minsalud.gov.co/sites/rid/Lists/BibliotecaDigital/RIDE/VS/ED/ PSP/PDSP.pdf

54. Colombia, Ministerio de Salud y Protección Social (MSPS); Instituto Nacional de Cancerología (INC). E.S.E. Plan decenal para el control del cáncer en Colombia 2012-2021. Bogotá: MSPS [internet]; 2012 [citado 2019 oct. 15]. Disponible en: https://www. minsalud.gov.co/Documentos\%20y\%20Publicaciones/Plan\%20 Decenal\%20para\%20el\%20Control\%20del $\% 20 \mathrm{C} \% \mathrm{C} 3 \%$ A 1 ncer. pdf

55. Bella F, Minicozzi P, Giacomin A, et al. Impact of diabetes on overall and cancer-specific mortality in colorectal cancer patients J Cancer Res Clin Oncol. 2013;139(8):1303-10. DOI: https://doi. org/10.1007/s00432-013-1439-8

56. Chen KH, Shao YY, Lin ZZ, et al. Type 2 diabetes mellitus is associated with increased mortality in Chinese patients receiving curative surgery for colon cancer. Oncologist. 2014;19(9):951-8. DOI: https://doi.org/10.1634/theoncologist.2013-0423

57. Ospina ML, Huertas JA, Montaño JI, et al. Observatorio Nacional de Cáncer Colombia. Rev. Fac. Nac. Salud Pública. 2015;33(2):262-76. DOI: http://dx.doi.org/10.17533/udea.rfnsp. v33n2a13

58. Colombia, Ministerio de Salud y Protección Social (MSPS), Instituto de Evaluación Tecnológica en Salud (IETS), Instituto Nacional de Cancerología (INC) E.S.E. Guía de práctica clínica (GPC) para la detección temprana, diagnóstico, tratamiento integral, seguimiento y rehabilitación del cáncer de colon y recto. 2.a ed. Bogotá: MSPS [internet]; 2017 [citado 2019 oct. 15]. http://gpc.minsalud.gov.co/gpc_sites/Repositorio/Conv_500/ GPC cancer colon/GPC Comple Ca Colon.pdf

59. León E, Gamboa Ó, Lozano T, et al. Estudio de costo de la enfermedad en pacientes con cáncer de colon y recto en
Colombia. Rev. Colom. Cancerol. 2013;17(4):184. DOI: https:// doi.org/10.1016/S0123-9015(13)70209-9

60. Vargas-Uricoechea H, Casas-Figueroa LA. Epidemiología de la diabetes mellitus en Sudámerica: la experiencia de Colombia. Clin Invest Arterioscler. 2016;28(5):245-56. DOI: https://doi. org/10.1016/j.arteri.2015.12.002

61. Ortegate MA, Sangiovanni S, Díaz MC, Aguilar J, García JI, Asencio H. Epidemiología de diabetes mellitus tipo 2 en la población colombiana y factores de riesgo que predisponen a la amputación de miembros inferiores. Revisión de la literatura. Salutem Scientia Spiritus [internet]. 2018 [citado 2020 ene. 27]; 4(1): 49-56. Disponible en: https://revistas.javerianacali.edu.co/ index.php/salutemscientiaspiritus/article/view/1937

62. Organización Mundial de la Salud (OMS). Perfiles de los países para la diabetes. Colombia. Ginebra: OMS [internet]; 2016 [citado 2019 oct. 15]. Disponible en: http://www.who.int/diabetes/ country-profiles/col_es.pdf?ua $=1$

63. Machado-Alba JE, Moncada-Escobar JC, Gaviria H. Quality and effectiveness of diabetes care for a group of patients in Colombia. Rev Panam Salud Publica [internet]. 2009 [citado 2020 ene. 27]; 26(6):529-35. Disponible en: https://www.ncbi.nlm.nih.gov/ pubmed/20107707

64. Ministerio de Salud y Protección Social (MSPS); Departamento Administrativo de Ciencia, Tecnología e Innovación (Colciencias). Guía de práctica clínica para el diagnóstico, tratamiento y seguimiento de la diabetes mellitus tipo 2 en la población mayor de 18 años. Bogotá: MSPS [internet]; 2016 [citado 2019 oct. 15]. Disponible en: http://gpc.minsalud.gov.co/gpc sites/Repositorio/ Conv_637/GPC_diabetes/DIABETES_TIPO_2_COMPLETA.pdf 